\title{
The helminth glycoprotein omega-1 improves metabolic homeostasis in obese mice through type 2 immunity-independent inhibition of food intake
}

\author{
Hendrik J.P. van der Zande ${ }^{1} \quad$ Michael A. Gonzalez ${ }^{2} \quad$ Karin de Ruiter $^{1}$ | \\ Ruud H.P. Wilbers ${ }^{3}$ | Noemí García-Tardón ${ }^{1}$ ｜ Mariska van Huizen ${ }^{1}$ | Kim van Noort ${ }^{3}$ \\ Leonard R. Pelgrom $^{1}$ ｜ Joost M. Lambooij ${ }^{1}$ | Anna Zawistowska-Deniziak ${ }^{1,4}$ | \\ Frank Otto $^{1}$ | Arifa Ozir-Fazalalikhan' ${ }^{1}$ Danny van Willigen ${ }^{5}$ | Mick Welling | \\ Jordan Poles $^{2}$ | Fijs van Leeuwen ${ }^{5}$ | Cornelis H. Hokke ${ }^{1}$ | Arjen Schots ${ }^{3}$ | \\ Maria Yazdanbakhsh $^{1}$ | P'ng Loke ${ }^{2,6}$ | Bruno Guigas ${ }^{1}$
}

${ }^{1}$ Department of Parasitology, Leiden University Medical Center, Leiden, The Netherlands

${ }^{2}$ Department of Microbiology, New York University School of Medicine, New York, NY, USA

${ }^{3}$ Department Laboratory of Nematology, Wageningen University and Research, Wageningen, The Netherlands

${ }^{4}$ Witold Stefański Institute of Parasitology, Polish Academy of Sciences, Warsaw, Poland

${ }^{5}$ Interventional Molecular Imaging Laboratory, Department of Radiology, Leiden University Medical Center, Leiden, The Netherlands

${ }^{6}$ Laboratory of Parasitic Diseases, National Institute of Allergy and Infectious Diseases, National Institutes of Health, Bethesda, MD, USA

\section{Correspondence}

Bruno Guigas, Department of Parasitology, Leiden University Medical Center, P.O. Box

\begin{abstract}
Type 2 immunity plays an essential role in the maintenance of metabolic homeostasis and its disruption during obesity promotes meta-inflammation and insulin resistance. Infection with the helminth parasite Schistosoma mansoni and treatment with its soluble egg antigens (SEA) induce a type 2 immune response in metabolic organs and improve insulin sensitivity and glucose tolerance in obese mice, yet, a causal relationship remains unproven. Here, we investigated the effects and underlying mechanisms of the T2 ribonuclease omega-1 $(\omega 1)$, one of the major $S$ mansoni immunomodulatory glycoproteins, on metabolic homeostasis. We show that treatment of obese mice with plant-produced recombinant $\omega 1$, harboring similar glycan motifs as present on the native molecule, decreased body fat mass, and improved systemic insulin sensitivity and glucose tolerance in a time- and dose-dependent manner. This effect was associated with an increase in white adipose tissue (WAT) type $2 \mathrm{~T}$ helper cells, eosinophils, and alternatively activated macrophages, without affecting type 2 innate lymphoid cells. In contrast to SEA, the metabolic effects of $\omega 1$ were still observed in obese STAT6-deficient mice with impaired type 2 immunity, indicating that its metabolic effects are independent of the type 2 immune response. Instead, we found that $\omega 1$ inhibited food intake, without affecting locomotor activity,
\end{abstract}

\footnotetext{
Abbreviations: AAM, alternatively activated macrophage; ALAT, alanine aminotransferase; BAT, brown adipose tissue; DC, dendritic cell; EE, energy expenditure; GTT, glucose tolerance test; HEK, human embryonic kidney; HFD, high-fat diet; HOMA-IR, homeostatic model assessment of insulin resistance; IFN- $\gamma$, interferon gamma; IL, interleukin; ILC, innate lymphoid cell; ITT, insulin tolerance test; LBM, lean body mass; LFD, low-fat diet; NAFLD, nonalcoholic fatty liver disease; NASH, nonalcoholic steatohepatitis; $\mathrm{pLe}^{\mathrm{X}}-\omega 1$, recombinant omega-1 from Le $\mathrm{x}^{\mathrm{X}}$-glyco-engineered Nicotiana benthamiana plants; PMA, phorbol myristate acetate; pWT- $\omega 1$, recombinant omega-1 from wild-type $N$ benthamiana plants; SEA, Schistosoma mansoni soluble egg antigens; STAT, signal transducer and activator of transcription; SVF, stromal vascular fraction; Th, T helper; TNF, tumor necrosis factor; UCP1, uncoupling protein 1; WAT, white adipose tissue; WT, wild-type.
}

This is an open access article under the terms of the Creative Commons Attribution-NonCommercial-NoDerivs License, which permits use and distribution in any medium, provided the original work is properly cited, the use is non-commercial and no modifications or adaptations are made.

(C) 2021 The Authors. The FASEB Journal published by Wiley Periodicals LLC on behalf of Federation of American Societies for Experimental Biology. 
9600, Postal zone P4-P, 2300 RC Leiden, the Netherlands.

Email: b.g.a.guigas@1umc.nl

\section{Funding information}

ZonMw (Netherlands Organisation for Health Research and Development), Grant/ Award Number: 91214131; Nederlandse Organisatie voor Wetenschappelijk Onderzoek (NWO), Grant/Award Number: 022.006.010; Division of Intramural Research, National Institute of Allergy and Infectious Diseases (DIR, NIAID), Grant/ Award Number: AI130945; Division of Intramural Research, National Institute of Allergy and Infectious Diseases (DIR, NIAID), Grant/Award Number: AI133977; HHS I NIH I National Heart, Lung, and Blood Institute (NHLBI), Grant/Award Number: HL084312; U.S. Department of Defense (DOD), Grant/Award Number: W81XWH-16-1-0256
WAT thermogenic capacity or whole-body energy expenditure, an effect also occurring in leptin receptor-deficient obese and hyperphagic $d b / d b$ mice. Altogether, we demonstrate that while the helminth glycoprotein $\omega 1$ can induce type 2 immunity, it improves whole-body metabolic homeostasis in obese mice by inhibiting food intake via a STAT6-independent mechanism.

\section{K E Y W O R D S}

helminths, immunometabolism, insulin sensitivity, macrophages, type 2 immunity

\section{\begin{tabular}{l|l}
1 & INTRODUCTION
\end{tabular}}

Obesity is associated with chronic low-grade inflammation in metabolic organs. ${ }^{1}$ This so-called meta-inflammation plays a prominent role in the etiology of insulin resistance and type 2 diabetes, ${ }^{1-3}$ and is associated with increased numbers of pro-inflammatory macrophages, notably in white adipose tissue (WAT) ${ }^{4}$ and liver. ${ }^{5}$ In WAT, these macrophages mainly originate from newly recruited blood monocytes that differentiate into pro-inflammatory macrophages upon entering the inflammatory milieu ${ }^{4}$ and/ or being activated by elevated local concentration of free fatty acids. ${ }^{6}$ These pro-inflammatory macrophages produce cytokines, such as tumor necrosis factor (TNF) and interleukin 1-beta (IL-1 $\beta$ ), which directly inhibit canonical insulin signaling [as reviewed in Ref. 2] and contribute to tissue-specific insulin resistance and whole-body metabolic dysfunctions. In the liver, activation of Kupffer cells, the tissue-resident macrophages, promote the recruitment of pro-inflammatory monocytes and neutrophils which trigger hepatic inflammation and insulin resistance through the production of pro-inflammatory cytokines and elastase, respectively. ${ }^{5,7,8}$ In contrast, a type 2 cytokine environment predominates in lean metabolic tissues under homeostatic conditions, notably in WAT where IL-4, IL-5, and IL-13 produced by type 2 innate lymphoid cells (ILC2s), T helper 2 (Th2) cells, and/or eosinophils promote alternatively activated macrophages (AAM). ${ }^{9,10}$ According to the current paradigm, AAMs are the final effector cells of this type 2 immune response, contributing to the maintenance of WAT insulin sensitivity by underlying molecular mechanism(s) that are still largely unknown. ${ }^{2,11}$
Parasitic helminths are the strongest natural inducers of type 2 immunity. ${ }^{12}$ Interestingly, several studies have reported an association between helminth-induced type 2 immunity and improved whole-body metabolic homeostasis in both humans and rodents [Ref. 13 and as reviewed in Ref. 11]. We also showed that chronic treatment with Schistosoma mansoni soluble egg antigens (SEA) promoted eosinophilia, Th2 cells, type 2 cytokines expression, and AAMs in WAT, and improved both tissue-specific and systemic insulin sensitivity in obese mice. ${ }^{14}$ SEA drives dendritic cell (DC)-mediated Th2 skewing at least partly through glycosylated molecules [Ref. 15 and reviewed in Ref. 16], particularly the T2 RNase glycoprotein omega- $\left.1 \omega 1^{17,18}\right]$. Interestingly, acute treatment with human embryonic kidney 293 (HEK-293)-produced recombinant $\omega 1$ was recently shown to decrease body weight and improve whole-body glucose tolerance in obese mice, through ILC2-mediated type 2 immunity and induction of WAT beiging. ${ }^{19}$ In this study, the metabolic effect of $\omega 1$ was reported to be glycan-dependent, yet, we have previously shown that the glycosylation pattern of HEK-293-produced $\omega 1$ differs significantly from the $S$ mansoni native molecule, which notably harbors immunogenic Lewis- $\mathrm{X}\left(\mathrm{Le}^{\mathrm{X}}\right)$ glycan motifs. ${ }^{18,20}$ By exploiting the flexible N-glycosylation machinery of Nicotiana benthamiana plants, we successfully produced large amounts of recombinant $\omega 1$ glycosylation variants, either carrying $\mathrm{Le}^{\mathrm{X}}$ motifs on one of its glycan branches or not. ${ }^{21}$

In the present study, we investigate the effects and underlying immune-dependent mechanisms of both SEA and two plant-produced $\omega 1$ glycovariants on whole-body metabolic homeostasis in obese mice. Remarkably, we demonstrate that while SEA improved metabolic homeostasis in obese 
mice through a STAT6-dependent type 2 immune response, recombinant $\mathrm{pLe}^{\mathrm{X}}-\omega 1$ did so independent of its type 2 immunity-inducing capacity, by reducing food intake in a leptin receptor-independent manner.

\section{2 | METHODS}

\subsection{Animals, diet, and treatment}

All mouse experiments were performed in accordance with the Guide for the Care and Use of Laboratory Animals of the Institute for Laboratory Animal Research and have received approval from the university Ethical Review Board (Leiden University Medical Center, Leiden, The Netherlands; DEC12199) or the Institutional Animal Care and Use Committee (IACUC, New York University School of Medicine, New York, USA; protocol ID IA16-00864). All mice were housed in a temperaturecontrolled room with a 12 hour light-dark cycle with ad libitum access to food and tap water. Group randomization was systematically performed before the start of each experiment, based on body weight, fat mass, and fasting plasma glucose levels. At the end of the experiment, mice were sacrificed through an overdose of ketamine/xylazine.

About 8-10 weeks old male wild-type (WT) and 7 weeks old male $d b / d b$ mice, both on C57BL6/J background, were purchased from Envigo (Horst, The Netherlands) and housed at Leiden University Medical Center. WT mice were fed a low-fat diet (LFD, 10\% energy derived from fat, D12450B, Research Diets, Wijk bij Duurstede, The Netherlands) or a high-fat diet (HFD, 45\% energy derived from fat, D12451) for 12 weeks, and $d b / d b$ mice were fed a chow diet (RM3 (P), Special Diet Services, Witham, UK) throughout the experimental period.

About 8-10 weeks old male wild-type (WT), Stat6 ${ }^{-/-}$ mice, and Rosa26 ${ }^{\text {LoxP-STOP-LoxP-tdTomato/+ }}$ (Rosa26 $\left.6^{\text {tdTomato }}\right)$, were purchased from The Jackson Laboratory (Bar Harbor, ME, USA), and $\mathrm{Cx} 3 \mathrm{crl} \mathrm{C}^{\mathrm{CreERT2}-\mathrm{IRES}-\mathrm{YFP} /+}\left(\mathrm{Cx} 3 \mathrm{crl} \mathrm{C}^{\mathrm{CrER}}\right)$ mice, all on C57BL/6J background, were generously provided by Dr Dan Littman (Skirball Institute, New York University Medical Center). Mice were housed at New York University School of Medicine, and either put on a HFD (60\% energy derived from fat; D12492; Research Diets, New Brunswick, NJ, USA) or LFD (10\% energy derived from fat; D12450J; Research Diets) for 10 weeks. To exclude effects of genotype-dependent microbiota differences on metabolic and immunological outcomes, the beddings of WT and Stat $6^{-1-}$ mice were frequently mixed within similar diet groups throughout the run-in period. At 14 days and 7 days before sacrifice, $C x 3 \mathrm{crl} 1^{\mathrm{CreER}} \operatorname{Rosa} 26^{\text {tdTomato }}$ reporter mice received an oral gavage with tamoxifen to label monocytes.

SEA was prepared as described previously. ${ }^{22}$ Recombinant $\omega 1$ was produced in $N$ benthamiana plants through transient expression of $\omega 1$ alone (pWT- $\omega 1$ ) or $\omega 1$ in combination with exogenous glycosyltransferases to yield $\mathrm{Le}^{\mathrm{X}}$ glycan motifs
(pLe $\left.{ }^{\mathrm{X}}-\omega 1\right)$, as described previously. ${ }^{21}$ SEA, pWT/pLe ${ }^{\mathrm{X}}-\omega 1$ (10$50 \mu \mathrm{g}$ ) or vehicle control (sterile-filtered PBS) were injected i.p. every 3 days for 1 or 4 weeks, as indicated in the legends of the figures. For fast-refeeding experiments, WT HFD-fed mice received an i.p. injection of $50 \mu \mathrm{g} \mathrm{pLe}^{\mathrm{X}}-\omega 1$ or vehicle control after an overnight fast (5PM-9AM), followed by refeeding and frequent measurements of food intake and body weight during 24 hours. For assessing the contribution of reduced food intake on the immunometabolic effects of $\mathrm{pLe}^{\mathrm{X}}-\omega 1$, WT HFD-fed mice were single-housed and, in a pair-fed group of PBS-injected mice, daily food availability was adjusted to the calorie intake of the $\mathrm{pLe}^{\mathrm{X}}-\omega 1$-treated group.

\subsection{Body composition and indirect calorimetry}

Body composition was measured by MRI using an EchoMRI (Echo Medical Systems, Houston, TX, USA). Groups of 4-8 mice with free access to food and water were subjected to individual indirect calorimetric measurements during the initiation of the treatment with recombinant $\omega 1$ for a period of 7 consecutive days using a Comprehensive Laboratory Animal Monitoring System (Columbus Instruments, Columbus, $\mathrm{OH}$, USA). Before the start of the measurements, single-housed animals were acclimated to the cages for a period of 48 hours. Feeding behavior was assessed by real-time food intake. Oxygen consumption and carbon dioxide production were measured at 15 minute intervals. Energy expenditure (EE) was calculated and normalized for lean body mass (LBM), as previously described. ${ }^{14}$ Spontaneous locomotor activity was determined by the measurement of beam breaks.

At sacrifice, visceral white adipose tissue (epididymal; eWAT), subcutaneous white adipose tissue (inguinal; iWAT), supraclavicular brown adipose tissue (BAT), and liver were weighed and collected for further processing and analyses.

\subsection{Isolation of stromal vascular fraction from adipose tissue}

eWAT was collected at sacrifice after a 1 minute perfusion with PBS through the heart left ventricle and digested as described previously. ${ }^{14}$ In short, collected tissues were minced and incubated for 1 hour at $37^{\circ} \mathrm{C}$ in an agitated incubator $(60 \mathrm{rpm})$ in HEPES buffer ( $\mathrm{pH} 7.4$ ) containing $0.5 \mathrm{~g} / \mathrm{L}$ collagenase type I from Clostridium histolyticum (Sigma-Aldrich, Zwijndrecht, The Netherlands) and 2\% (w/v) dialyzed bovine serum albumin (BSA, fraction V; Sigma-Aldrich). The disaggregated adipose tissue was passed through a $100 \mu \mathrm{m}$ cell strainer that was washed with PBS supplemented with 2.5 of mM EDTA and $5 \%$ of FCS. After centrifugation $(350 \mathrm{~g}, 10 \mathrm{~min}$ at room temperature), the supernatant was discarded and the pellet was 
treated with erythrocyte lysis buffer $\left(0.15 \mathrm{M} \mathrm{NH}_{4} \mathrm{Cl} ; 1 \mathrm{mM}\right.$ $\left.\mathrm{KHCO}_{3} ; 0.1 \mathrm{mM} \mathrm{Na} 2 \mathrm{EDTA}\right)$. Cells were next washed with $\mathrm{PBS} / \mathrm{EDTA} / \mathrm{FCS}$, and counted manually.

\subsection{Isolation of leukocytes from liver tissue}

Livers were collected and digested as described previously. ${ }^{14}$ In short, livers were minced and incubated for 45 minutes at $37^{\circ} \mathrm{C}$ in RPMI 1640 + GlutaMAX (Life Technologies, Bleiswijk, The Netherlands) containing $1 \mathrm{mg} / \mathrm{mL}$ collagenase type IV from $C$ histolyticum, $2000 \mathrm{U} / \mathrm{mL}$ DNase (both Sigma-Aldrich), and $1 \mathrm{mM} \mathrm{CaCl}{ }_{2}$. The digested liver tissues were passed through a $100 \mu \mathrm{m}$ cell strainer that was washed with PBS/EDTA/FCS. Following centrifugation $\left(530 \mathrm{~g}, 10\right.$ minutes at $\left.4{ }^{\circ} \mathrm{C}\right)$, the supernatant was discarded, after which the pellet was resuspended in PBS/EDTA/FCS and centrifuged at $50 \mathrm{~g}$ to pellet hepatocytes ( 3 minutes at $4^{\circ} \mathrm{C}$ ). Next, supernatants were collected and pelleted $\left(530 \mathrm{~g}, 10\right.$ minutes at $\left.4^{\circ} \mathrm{C}\right)$. The cell pellet was first treated with erythrocyte lysis buffer and next washed with PBS/EDTA/ FCS. CD $45^{+}$leukocytes were isolated using LS columns and CD45 MicroBeads (35 $\mu \mathrm{L}$ beads per liver, Miltenyi Biotec) according to manufacturer's protocol and counted manually.

\subsection{Processing of isolated immune cells for flow cytometry}

For analysis of macrophage and lymphocyte subsets, both WAT stromal vascular cells and liver leukocytes were stained with the live/dead marker Aqua (Invitrogen, Bleiswijk, The Netherlands) or Zombie-UV (Biolegend, San Diego, CA, USA), fixed with either $1.9 \%$ of formaldehyde (SigmaAldrich) or the eBioscience FOXP3/Transcription Factor Staining Buffer Set (Invitrogen), and stored in FACS buffer (PBS, $0.02 \%$ sodium azide, $0.5 \% \mathrm{FCS}$ ) at $4^{\circ} \mathrm{C}$ in the dark until subsequent analysis. For analysis of cytokine production, isolated cells were cultured for 4 hours in culture medium in the presence of $100 \mathrm{ng} / \mathrm{mL}$ phorbol myristate acetate (PMA), $1 \mu \mathrm{g} / \mathrm{mL}$ ionomycin, and $10 \mu \mathrm{g} / \mathrm{mL}$ Brefeldin A (all from Sigma-Aldrich). After 4 hours, cells were washed with PBS, stained with Aqua, and fixed as described above.

\subsection{Flow cytometry}

For analysis of CD4 T cells and innate lymphoid cell (ILC) subsets, SVF cells were stained with antibodies against B220 (RA3-6B2), CD11b (M1/70), CD3 (17A2), CD4 (GK1.5), NK1.1 (PK136) and Thy1.2 (53-2.1; eBioscience), CD11c (HL3) and GR-1 (RB6-8C5; both BD Biosciences, San Jose, CA, USA), and CD45.2 (104; eBioscience, Biolegend or Tonbo Biosciences, San Diego, CA, USA). CD4 T cells were identified as $\mathrm{CD} 45^{+}$Thy $1.2^{+}$Lineage $^{+} \mathrm{CD} 4^{+}$, and ILCs as $\mathrm{CD} 45^{+}$Thy $1.2^{+}$Lineage $^{-} \mathrm{CD} 4^{-}$cells, in which the lineage cocktail included antibodies against CD11b, CD11c, B220, GR-1, NK1.1, and CD3.

CD4 $\mathrm{T}$ cell subsets and cytokine production by ILCs were analyzed following permeabilization with either $0.5 \%$ of saponin (Sigma-Aldrich) or eBioscience FOXP3/Transcription Factor Staining Buffer Set. Subsets were identified using antibodies against CD11b, CD11c, GR-1, B220, NK1.1, CD3, CD45.2, CD4, Thy1.2, IL-4 (11B11), IL-13 (eBio13A), Foxp3 (FJK-16s; all eBioscience), IL-5 (TRFK5), and IFN- $\gamma$ (XMG1.2; both Biolegend).

For analysis of macrophages, eosinophils, monocytes, and neutrophils, cells were permeabilized as described above. Cells were then incubated with an antibody against YM1 conjugated to biotin (polyclonal; R\&D Systems, Minneapolis, MN, USA), washed, and stained with streptavidin-PerCP (BD Biosciences) or streptavidin-PerCP-Cy5.5 (Biolegend), and antibodies directed against CD45 (30-F11, Biolegend), CD45.2, CD11b, CD11c [HL3 (BD Biosciences) or N418 (Biolegend)], F4/80 (BM8; Invitrogen or Biolegend), Siglec-F (E50-2440; BD Biosciences), and Ly6C (HK1.4; Biolegend).

Mitochondrial mass, membrane potential, and total ROS were quantified by staining with MitoTracker Green ( $20 \mathrm{nM}$ ), Tetramethylrhodamine, Methyl Ester, Perchlorate (TMRM; $20 \mathrm{nM}$ ), and CM-H2DCFDA (5 $\mu \mathrm{M}$; all Invitrogen), respectively, for 30 minutes at $37^{\circ} \mathrm{C}$ before staining with other antibodies. For sorting adipose tissue macrophages, cells were stained with the live/dead marker Blue, followed by staining for surface markers. Macrophages were fluorescence-assisted cell sorted from adipose tissue on an Aria II cell sorter (BD Biosciences), by gating on singlet, live, CD $45.2^{+} \mathrm{Ly}^{-} \mathrm{C}^{-} \mathrm{CD}^{-}$ CD19 ${ }^{-}$NK1.1 $1^{-}$Siglec-F CD11b ${ }^{+}$F4/80 ${ }^{+}$tdTomato $^{+}$.

All cells were stained and measured within 4 days post fixation. Flow cytometry was performed using a FACSCanto or LSR-II (both BD Biosciences), and gates were set according to Fluorescence Minus One (FMO) controls. Representative gating schemes are shown in Figure S1 and all antibodies used are listed in Table S1.

\section{7 | Plasma analysis}

Blood samples were collected from the tail tip of $4 \mathrm{~h}$-fasted mice (food removed at $9 \mathrm{AM}$ ) using chilled paraoxon-coated capillaries. Fasting blood glucose level was determined using a Glucometer (Accu-Chek; Roche Diagnostics, Almere, The Netherlands) and plasma insulin level was measured using a commercial kit according to the instructions of the manufacturer (Chrystal Chem, Zaandam, The Netherlands). The homeostatic model assessment of insulin resistance (HOMA-IR) adapted to mice ${ }^{23}$ was calculated as ([glucose $(\mathrm{mg} / \mathrm{dL}) * 0.055] \times[$ insulin $(\mathrm{ng} / \mathrm{ml}) \times 172.1]) / 3857$, and used 
as a surrogate measure of whole-body insulin resistance. Plasma concentrations of alanine aminotransferase (ALAT) were measured using a Reflotron $®$ kit (Roche diagnostics) using a pool of plasma samples from each group $(n=4-6$ mice per group) in two separate experiments.

\subsection{Glucose, insulin, and pyruvate tolerance tests}

Whole-body glucose tolerance test (ipGTT) was performed at week 3 of treatment in 6 hours-fasted mice, as previously reported. ${ }^{14}$ In short, after an initial blood collection by tail bleeding $(\mathrm{t}=0)$, a glucose load ( $2 \mathrm{~g} / \mathrm{kg}$ total body weight of D-Glucose [Sigma-Aldrich]) was administered i.p., and blood glucose was measured at 20, 40, 60, and 90 minutes after glucose administration using a Glucometer. For $d b / d b$ mice, blood samples were collected at 0, 20, 40, and 90 minutes after glucose administration, and plasma glucose levels were measured using the hexokinase method (HUMAN, Wiesbaden, Germany).

Whole-body insulin tolerance test (ipITT) was performed at week 1 or week 3 of treatment in 4 hours-fasted mice, as described previously. ${ }^{14}$ In short, after an initial blood collection by tail bleeding $(\mathrm{t}=0)$, a bolus of insulin ( $1 \mathrm{U} / \mathrm{kg}$ (lean) body mass [NOVORAPID, Novo Nordisk, Alphen aan den Rijn, The Netherlands]) was administered i.p., and blood glucose was measured at 20, 40, 60, and 90 minutes after insulin administration using a Glucometer.

Whole-body pyruvate tolerance test (ipPTT) was performed at week 4 of treatment in overnight-fasted mice. In short, after an initial blood collection by tail bleeding $(t=0)$, a pyruvate load $(2 \mathrm{~g} / \mathrm{kg}$ total body weight of sodium pyruvate [Sigma-Aldrich]) was administered i.p. Blood glucose was measured at 20, 60, 90, and 120 minutes after pyruvate administration using a Glucometer.

\subsection{Western blot analysis}

A piece of liver, skeletal muscle, eWAT, and iWAT from mice that were sacrificed 15 minutes after an acute i.p. injection of insulin (1 U/kg lean body mass, NOVORAPID, Novo Nordisk, Alphen aan den Rijn, The Netherlands) was collected and immediately freeze-clamped. Snap-frozen samples $(\sim 50 \mathrm{mg})$ were lysed in ice-cold buffer containing: $50 \mathrm{mM}$ HEPES ( $\mathrm{pH} 7.6$ ), $50 \mathrm{mM} \mathrm{NaF}, 50 \mathrm{mM} \mathrm{KCl}$, $5 \mathrm{mM}$ NaPPi, $1 \mathrm{mM}$ EDTA, $1 \mathrm{mM}$ EGTA, $1 \mathrm{mM}$ DTT, $5 \mathrm{mM} \beta$-glycerophosphate, $1 \mathrm{mM}$ sodium vanadate, $1 \%$ NP40, and protease inhibitors cocktail (Complete, Roche, Mijdrecht, The Netherlands). Western blots were performed as previously described. ${ }^{24}$ The primary antibodies used were pSer473-PKB (\#9271, Cell Signaling Technology), PKB (\#4691, Cell Signaling Technology), IRb (sc-711, Santa Cruz Biotechnology), and HSP90 (sc-7947, Santa Cruz
Biotechnology). Bands were visualized by enhanced chemiluminescence and quantified using Image J (NIH, US).

\subsection{0 | RNA isolation and qRT-PCR}

RNA was extracted from snap-frozen adipose tissue and liver samples using Tripure RNA Isolation reagent (Roche Diagnostics). Total RNA (1 $\mu \mathrm{g})$ was reverse transcribed and quantitative real-time PCR was performed with SYBR Green Core Kit on a MyIQ thermal cycler (Bio-Rad) using specific primers sets (available on request). mRNA expression was normalized to ribosomal protein, large, P0 (RplP0) mRNA content and expressed as fold change compared to LFD-fed or HFD-fed mice as indicated, using the $\Delta \Delta \mathrm{CT}$ method.

\subsection{1 | Transcriptomic analysis by RNA sequencing}

RNA isolation from FACS-purified adipose tissue macrophages was done as described previously with the QIAGEN RNeasy micro kit (QIAGEN, Venlo, The Netherlands). ${ }^{25}$ Libraries were generated for each sample using the CelSeq2 protocol $^{26}$ and were sequenced on Illumina HiSeq. Reads were mapped by Bowtie2.3.1 ${ }^{27}$ to the mus musculus reference genome, and uniquely mapped indices were determined by HTSeq-counts. ${ }^{28}$ Gene read counts were normalized to $B 2 m$ and Rplp0. Transcriptome data were visualized with an MA-plot. Upregulated ( $\log _{2}$ fold change $>2$ vs HFD) and downregulated ( $\log _{2}$ fold change $<-2$ vs HFD) genes were visualized in a heat map using the online software tool Morpheus (https://software.broadinstitute.org/morpheus).

\subsection{Hepatic triglyceride content}

Liver lipids were extracted as previously described. ${ }^{29}$ Briefly, $\sim 50 \mathrm{mg}$ liver samples were homogenized in 10:1 (vol/wt) ice-cold methanol using a FastPrep-24 5GTM High Speed Homogenizer (MP Biomedicals, Santa Ana, CA, USA). Subsequently, lipids were extracted from the homogenate using methanol-chloroform $(1: 3 \mathrm{v} / \mathrm{v})$ extraction. Protein concentration of the homogenate was determined by Pierce BCA protein assay (Thermo Fisher Scientific). Triglyceride (TG) of the lipid extract were determined using a commercially available kit (Roche Diagnostics).

\subsection{3 | Histological analysis}

A piece of liver was fixed in $4 \%$ of formaldehyde (SigmaAldrich), paraffin-embedded, sectioned at $4 \mu \mathrm{m}$ and stained with Hematoxylin and Eosin (H\&E), or Sirius Red to 
visualize collagen. Six fields at 20x magnification (total area $1.68 \mathrm{~mm}^{2}$ ) were used for the analysis of hepatic steatosis in H\&E-stained sections. On Sirius Red-stained sections, fibrosis was scored on 10 fields at 40x magnification (total area $1.23 \mathrm{~mm}^{2}$ ) as absent (score 0), present in the perisinusoidal or periportal area (score 1), present in the perisinusoidal and periportal (score 2), bridging fibrosis (score 3 ) or cirrhosis (score 4) as described elsewhere. ${ }^{30}$

\subsection{Biodistribution of $\mathrm{pLe}^{\mathrm{X}}-\boldsymbol{\omega 1}$}

\subsection{1 | Synthesis of $\mathrm{N}_{3}-\mathrm{Cy} 5-\mathrm{pLe}^{\mathrm{X}}-\omega 1$}

DTPA-DBCO click chelate and $\mathrm{Cy} 5-\mathrm{N}_{3}$ dye were synthesized as described previously, ${ }^{31}$ with one deviation for Cy5- $\mathrm{N}_{3}$ synthesis being the use of 1-(5-carboxypentyl)-2,3,3trimethyl-3H-indol-1-ium-5-sulfonate instead of 1-(5-carboxypentyl)-2,3,3-trimethyl-3H-indol-1-ium. Cy5$\mathrm{N}_{3}$ molecular mass was calculated to be 823.8 and found to be 824.0 using MALDI-TOF (Bruker, Leiderdorp, The Netherlands). To allow for $\mathrm{N}_{3}-\mathrm{Cy} 5-\mathrm{pLe}^{\mathrm{X}}-\omega 1$ formation, $200 \mu \mathrm{L}$ of phosphate buffer $(100 \mathrm{mM}, \mathrm{pH} 8.5)$ was added to $200 \mu \mathrm{g}(6.5 \mathrm{nmol}) \mathrm{pLe}^{\mathrm{X}}-\omega 1(1.9 \mathrm{mg} / \mathrm{mL}$ in PBS$)$, followed by $9.5 \mu \mathrm{L}(58.1 \mathrm{nmol})$ of $\mathrm{Cy} 5-\mathrm{N}_{3}(5 \mathrm{mg} / \mathrm{mL}$ stock solution in DMSO). After mixing for 60 minutes at room temperature, $90 \mu \mathrm{L}$ of PBS was added and the unreacted Cy5 was removed using a PD MiniTrap G-25 column (Merck KGaA, Darmstadt, Germany). The labeling ratio was estimated to be 0.9 dye/protein using UV/Vis spectroscopy (Nanodrop) and the eluate $(1 \mathrm{~mL})$ was stored at $7^{\circ} \mathrm{C}$ prior to use.

\subsection{2 | Radiolabeling of $\mathrm{pLe}^{\mathrm{X}}-\omega 1$}

DTPA-DBCO (2.6 nmol) was dissolved in $17.3 \mu \mathrm{L}$ of ammonium acetate buffer $(250 \mathrm{mM}, \mathrm{pH} 5.5)$, and $9.6 \mathrm{MBq}$ or 48.0 MBq of ${ }^{111} \mathrm{InCl}_{3}$ (370 MBq/mL, Mallinckrodt Medical, Petten, The Netherlands) was added for biodistribution or SPECT imaging, respectively. The mixture was shaken for 60 minutes at room temperature followed by addition of PBS (200 mM, pH 7.5). Of this mixture, 4.1 MBq or 20.7 MBq was added to $1.3 \mathrm{nmol}$ or $0.7 \mathrm{nmol}$ of $\mathrm{N}_{3}-\mathrm{Cy} 5-\mathrm{pLe}^{\mathrm{X}}-\omega 1$ and stirred overnight at room temperature to form ${ }^{111}$ In-DTPA-Cy5$\mathrm{pLe}^{\mathrm{X}}-\omega 1$. The reaction mixture was directly used for injection.

\subsection{3 | SPECT imaging}

Ten week HFD-fed mice were injected intraperitoneally with $10 \mu \mathrm{g}{ }^{111}$ In-DTPA-Cy5-pLe ${ }^{\mathrm{X}}-\omega 1$ (10 MBq/mouse). Twentyfour hours post injection, in vivo biodistribution was assessed after the animals were placed and fixed on a dedicated positioned bed of a three-headed U-SPECT-2 (MILabs, Utrecht, The Netherlands) under continuous 1\%-2\% of isoflurane anesthesia. Radioactivity counts from total body scans and a second one of a head and neck area were acquired for 20 minutes and images were reconstructed as described before. ${ }^{32}$ After imaging, mice were euthanized by an intraperitoneal injection of $0.25 \mathrm{~mL}$ Euthasol (ASTfarma, Oudewater, The Netherlands).

\subsection{4 | Biodistribution}

For biodistribution experiments, organs were harvested, weighed, and radioactivity was counted (Wizard2 2470 automatic gamma scintillation counter, PerkinElmer, Groningen, The Netherlands). Total injected dose was determined by counting full and empty syringes in a gamma counter (2470 automatic gamma counter, Perkin-Elmer), and data are represented as $\%$ injected dose per gram tissue $(\% \mathrm{ID} / \mathrm{g})$, which was calculated as follows: ((([MBq]tissue/[MBq]injected) $\times 100) / g$ tissue $)$.

\subsection{5 | Statistical analysis}

All data are presented as mean \pm standard error of the mean (SEM). Statistical analysis was performed using GraphPad Prism version 8 for Windows (GraphPad Software, La Jolla, CA, USA) with unpaired $t$ test, or either one-way or two-way analysis of variance (ANOVA) followed by Fisher's post hoc test. Differences between groups were considered statistically significant at $P<.05$.

\section{3 | RESULTS}

\subsection{S mansoni soluble egg antigens (SEA) improve metabolic homeostasis in obese mice by a STAT6-dependent mechanism}

In order to investigate the role of type 2 immunity in the beneficial metabolic effects of SEA, we used mice deficient for STAT6 $\left(\right.$ Stat6 $\left.^{-/}\right)$, a key transcription factor involved in signature type 2 cytokines interleukin (IL)-4/IL-13 signaling and maintenance of $\mathrm{Th} 2$ effector functions. ${ }^{33,34}$ In line with previous studies, ${ }^{14,35}$ we treated HFD-fed obese WT and Stat6 $^{-/-}$with SEA for 4 weeks (Figure 1A). We confirmed that chronic treatment with SEA increased IL-5 and IL-13expressing Th2 cells (Figure 1B), eosinophils (Figure 1C), and $\mathrm{YM}^{+}{ }^{+} \mathrm{AAMs}$ (Figure 1D) in WAT from HFD-fed obese WT mice while, as expected, this type 2 immune response was abrogated in Stat6 $^{-/-}$mice. SEA slightly reduced body weight (Figure 1E) and similarly affected body composition 
(Figure S2A) in both WT and Stat6 ${ }^{-/}$obese mice, without affecting food intake (Figure 1F). In line with our previous study, we showed that SEA reduced fasting plasma insulin levels (Figure S2C) and HOMA-IR (Figure 1G), and improved whole-body glucose tolerance in WT obese mice (Figure 1H,J). Strikingly, this beneficial metabolic effect was completely abolished in Stat $6^{-1-}$ mice (Figure 1I,J), indicating that SEA improves whole-body metabolic homeostasis in obese mice through STAT6-mediated type 2 immunity.

\section{2 | Plant-produced recombinant $\omega 1$ glycovariants increase adipose tissue Th2 cells, eosinophils, and alternatively activated macrophages, without affecting innate lymphoid cells}

One of the major type 2 immunity-inducing molecules in SEA is the T2 ribonuclease glycoprotein $\omega 1 .^{18}$ To study the effect of $\omega 1$ on metabolic homeostasis and the role of its immunomodulatory glycans, we generated two recombinant glycosylation variants using glycol-engineered $N$ benthamiana plants: one carrying wild-type plant glycans (pWT- $\omega 1)$ and one harboring terminal $\mathrm{Le}^{\mathrm{X}}$ motifs $\left(\mathrm{pLe}^{\mathrm{X}}-\omega 1^{21}\right)$. For both $\omega 1$ glycovariants, 4 weeks treatment markedly increased WAT CD4 T cells in HFD-fed obese mice, with pWT- $\omega 1$ being slightly more potent than $\mathrm{pLe}^{\mathrm{X}}-\omega 1$, while total ILCs were unaffected (Figure 2A,B). Interestingly, a specific increase in WAT IL-5 and IL-13-expressing Th2 cells was seen for both $\omega 1$ glycovariants, while the other CD4 T cell subsets, that is, regulatory T cells (Treg) and Th1 cells, were not affected (Figure 2C). In addition, we confirmed that HFD reduced WAT IL- $5^{+} / \mathrm{IL}-13^{+}$ILC2s, as previously reported, ${ }^{9}$ an effect that was even further pronounced with $\omega 1$ glycovariants (Figure 2D). The type 2 cytokines IL-5 and IL-13 produced by either ILC2s and/or Th2 cells have been reported to maintain WAT eosinophils. ${ }^{9}$ Congruent with our data on Th2 cells, we found a potent increase in WAT eosinophils upon $\omega 1$ treatment that was of similar extent for both glycovariants (Figure 2E). Finally, both pWT- $\omega 1$ and $\mathrm{pLe}^{\mathrm{X}}-\omega 1$ increased $\mathrm{WAT} \mathrm{YM1}^{+}$AAMs while obesity-associated CD11c ${ }^{+}$macrophages were not affected (Figure $2 F, G$ ). This $\omega 1$-induced WAT type 2 immunity was dose-dependent (Figure S3) and already observed after 1 week of treatment, when ILC2s were also not affected (Figure S4).

AAMs are considered the effector cells of WAT type 2 immunity in the maintenance of tissue insulin sensitivity, ${ }^{2}$ although the mechanisms are not fully understood. Monocyte-derived macrophages can irreversibly be labeled upon tamoxifen administration in $\mathrm{Cx} 3 \mathrm{crl} \mathrm{C}^{\text {CreERT2-IRES-EYFP }}$ Rosa $26^{\text {LoxP-stop-LoxP-tdTomato }}\left(\mathrm{Cx} 3 \mathrm{crl} \mathrm{CrER}^{\mathrm{CreE}} \mathrm{Rosa} 26^{\text {tdTomato }}\right)$ mice, as described elsewhere. ${ }^{25}$ In order to characterize newly recruited, $\omega 1$-induced adipose tissue macrophages (ATMs) during obesity, we performed RNA sequencing
(A)

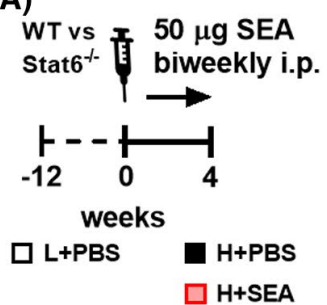

(F)

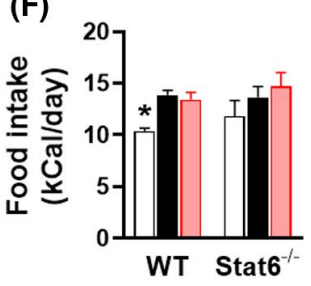

(B)

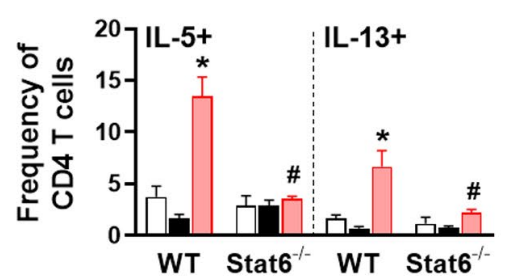

(G)

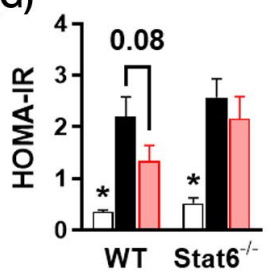

(C)

(H)

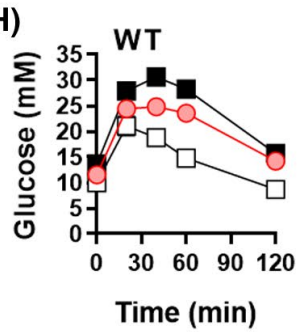

(D)

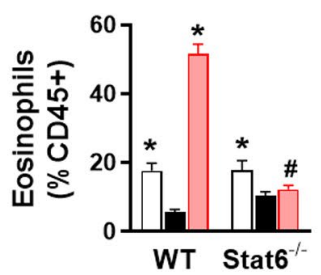

(I)

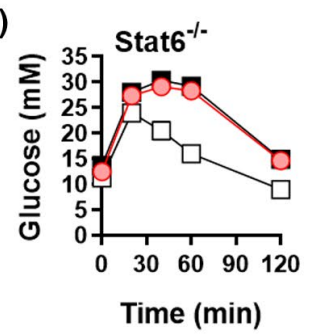

(E)
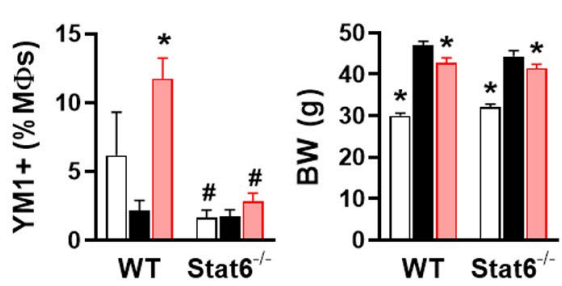

(J)

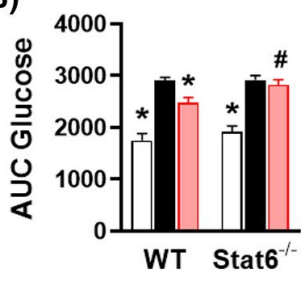

F I G U RE 1 S mansoni soluble egg antigens improve metabolic homeostasis in obese mice by a STAT6-dependent mechanism. WT and $\mathrm{Stat}^{-/-}$mice were fed a LFD (white bars) or a HFD for 12 weeks and next received intraperitoneal injections of PBS (black bars) or $50 \mu \mathrm{g} S$ mansoni soluble egg antigens (SEA; red bars) every 3 days for 4 weeks (A). At sacrifice, epididymal WAT was collected and SVF was isolated and analyzed by flow cytometry. The complete gating strategy is shown in Figure S1. Frequencies of IL-5 and IL-13-expressing Th2 cells (B) in WAT were determined after PMA/ionomycin/Brefeldin A restimulation. Abundances of eosinophils (C) and YM1 ${ }^{+}$macrophages (AAMs; D) were determined. Body weight (E) was measured after 4 weeks of treatment. Food intake (F) was monitored throughout the experimental period. HOMA-IR (G) was calculated using fasting blood glucose and plasma insulin levels at week 4. An i.p. glucose tolerance test was performed at week 3. Blood glucose levels were measured at the indicated time points (H-I) and the AUC of the glucose excursion curve was calculated (J). Data shown are a pool of two independent experiments. Results are expressed as means \pm SEM. ${ }^{*} P<.05$ vs HFD, ${ }^{\#} P<.05$ vs WT (n $=9-12$ mice per group) 

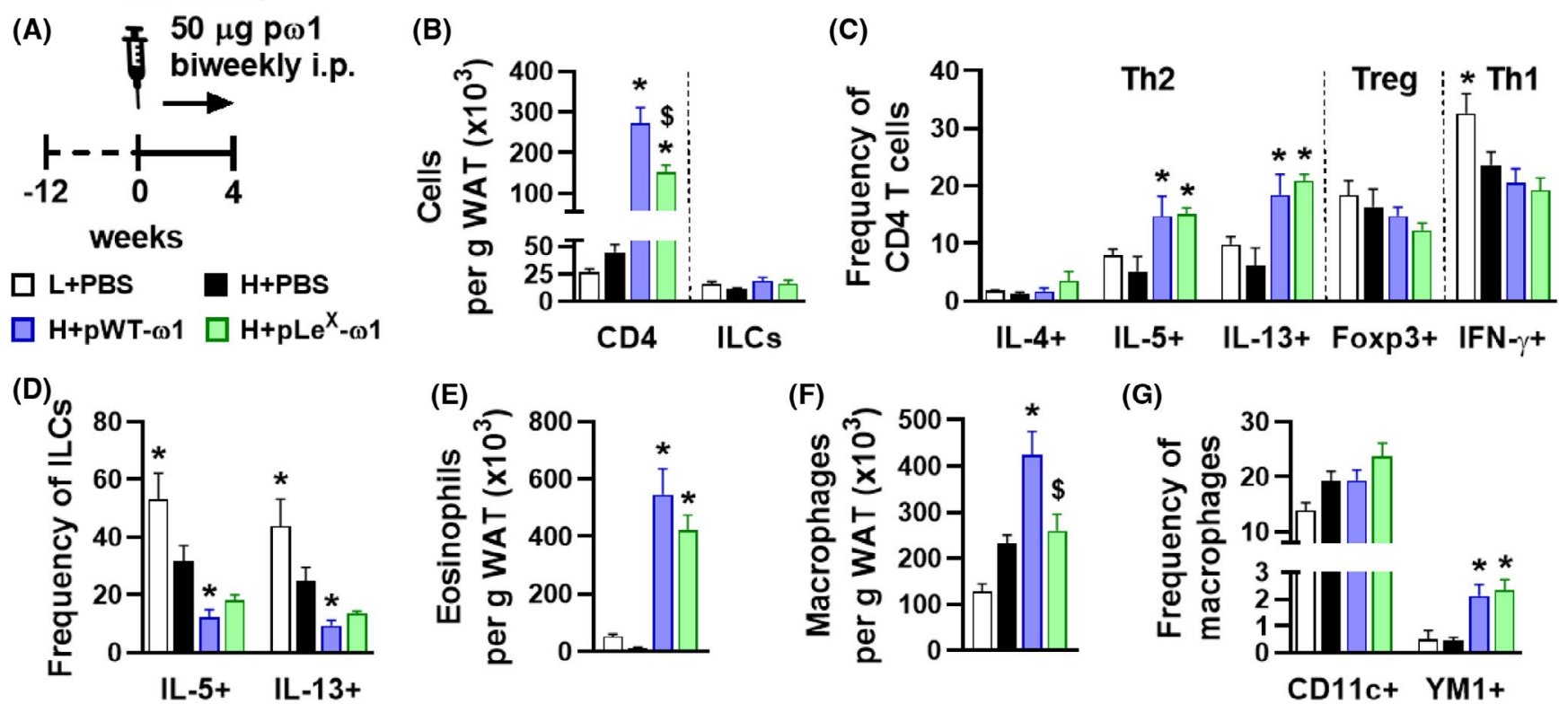

F I G U RE 2 Plant-produced recombinant $\omega 1$ glycovariants increase adipose tissue Th2 cells, eosinophils, and alternatively activated macrophages, without affecting innate lymphoid cells. Mice were fed a LFD (white bars) or a HFD for 12 weeks, and next received intraperitoneal injections of PBS (black bars) or either $50 \mu \mathrm{g}$ pW- $\omega 1$ (blue bars) or $50 \mu \mathrm{g} \mathrm{pLe} \mathrm{X}^{\mathrm{X}} \omega 1$ (green bars) every 3 days during 4 weeks (A). At the end of the experiment, eWAT was collected, processed, and analyzed as described in the legend of Figure 1. Numbers of CD4 T cells, ILCs (B), eosinophils (E), and macrophages (F) per gram tissue were determined. Frequencies of CD4 T helper subsets (C) and cytokine-expressing ILCs (D) were determined. Percentages of CD11 $\mathrm{c}^{+} \mathrm{YM} 1^{-}$and $\mathrm{CD} 11 \mathrm{c}^{-} \mathrm{YM} 1^{+}$macrophages $(\mathrm{G})$ were measured. Data shown are a pool of at least two independent experiments. Results are expressed as means \pm SEM. ${ }^{*} P<.05$ vs HFD, ${ }^{\$} P<.05$ vs pWT- $\omega 1$ ( $\mathrm{n}=6-19$ mice per group in B, E-G, and 3-9 mice per group in $\mathrm{C}$ and $\mathrm{D}$ )

on FACS-sorted tdTomato ${ }^{+}$macrophages from eWAT SVF of obese $\mathrm{Cx} 3 \mathrm{crl}^{\mathrm{CreER}}$ Rosa26 $6^{\text {tdTomato }}$ mice that were treated with PBS or $\mathrm{pLe}^{\mathrm{X}}-\omega 1$, the glycovariant that resembles native $\omega 1$ most (Figure S5A). Genes associated with alternative activation, for example, Tmem26, Slc7a2, Chil3, and $\operatorname{Arg} 1$, were upregulated $\left(\log _{2} \mathrm{FC}>2\right)$ in ATMs from $\mathrm{pLe}^{\mathrm{X}}-\omega 1$-treated mice as compared to controls, while genes associated with pro-inflammatory or obesity-associated macrophages, for example, $\operatorname{Igfbp} 7, \mathrm{Cxcl12}, \mathrm{Bgn}, \mathrm{Dcn}$, and Cd86, were downregulated $\left(\log _{2} \mathrm{FC}<-2\right.$; Figure S5B,C). Macrophage function is increasingly recognized to be supported by their metabolism to meet energy demands, and as such, AAMs display increased oxidative phosphorylation. ${ }^{36}$ In PD-L2 ${ }^{+}$WAT macrophages (Figure S5D), $\mathrm{pLe}^{\mathrm{X}}-\omega 1$ indeed increased mitochondrial mass, while displaying decreased mitochondrial membrane potential and similar total reactive oxygen species production (Figure S5E-G), a metabolic phenotype in line with alternative macrophage activation.

Similar to WAT, maintenance of insulin sensitivity in the liver is also associated with type 2 immunity, ${ }^{37}$ whereas obesity-driven activation of Kupffer cells increases the recruitment of pro-inflammatory monocytes and triggers hepatic insulin resistance. ${ }^{2,7}$ In our conditions, while $\omega 1$ glycovariants increased Th2 cells in the liver, we did not find alternative activation of Kupffer cells (Figure S6A-D). Instead, $\omega 1$ glycovariants increased the number of $\mathrm{CD} 11 \mathrm{c}^{+}$ pro-inflammatory Kupffer cells (Figure S6D), hepatic expression of pro-inflammatory cytokines $C c l 2$, $T n f$, and $I l 1 b$ (Figure S6E), and newly recruited monocytes (Figure S6F), with a more potent effect in $\mathrm{pLe}^{\mathrm{X}}-\omega 1$-treated mice. Taken together, these data indicate that both $\omega 1$ glycovariants potently induce type 2 immunity in obese mice, triggering an alternative activation profile in WAT, but not liver macrophages.

\section{3 $\quad \omega 1$ glycovariants reduce body weight, fat mass and food intake, and improve whole- body metabolic homeostasis in obese mice}

We next investigated the metabolic effects of $\omega 1$ glycovariants and showed that they both induced a rapid and gradual body weight loss in HFD-fed mice (Figure 3A,B), which was exclusively due to a decrease in fat mass (Figure 3C). The $\omega 1$ glycovariants significantly reduced visceral eWAT mass, but had no or only marginal effects on subcutaneous iWAT, brown adipose tissue (BAT) and liver mass (Figure $\mathrm{S} 2 \mathrm{D})$. This reduction in fat mass was associated with smaller adipocytes (Figure S7A,B), reduced leptin expression (Figure S7C,D), and opposite changes in expression of pro-inflammatory (decrease in Itgax) and alternatively activated (increase in Argl) canonical macrophage markers in both eWAT and iWAT (Figure S7C,D). However, 
the lower fat mass gain was clearly not due to increased WAT beiging, as $\omega 1$ glycovariants neither increased expression of thermogenic gene markers (Ucp1, Cox $8 b$, and Cidea) in both eWAT and iWAT from obese mice (Figure S7C,D), nor whole-body energy expenditure (Figure S7E). In addition, $\omega 1$ glycovariants did not affect hepatic steatosis (Figure S6G-I) but increased the expression of fibrotic gene markers (Figure S8A), without detectable collagen accumulation (Figure S8B,C). An increase in circulating alanine transaminase levels was also observed (Figure S8D), indicating that $\omega 1$ may also have some cytotoxic effects in the liver, as previously reported. ${ }^{38,39}$

Interestingly, we found that both $\omega 1$ glycovariants induced a significant decrease in food intake (Figure 3D,E), while locomotor activity was not affected (Figure 3F-G). Treatment with both $\omega 1$ glycovariants significantly reduced fasting blood
(A)

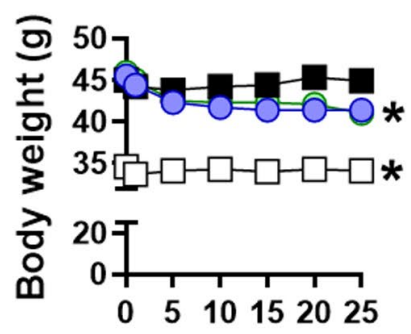

(D) Time (days)

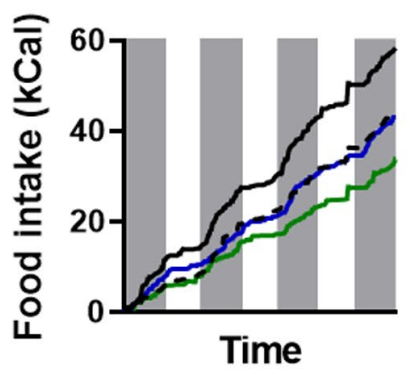

(H)

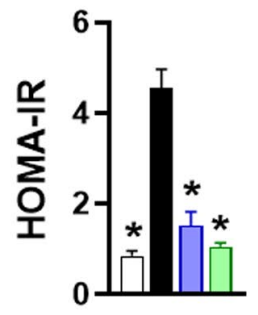

(M)

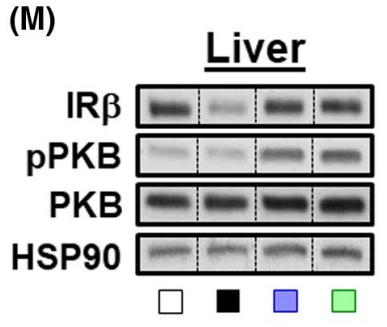

(I)

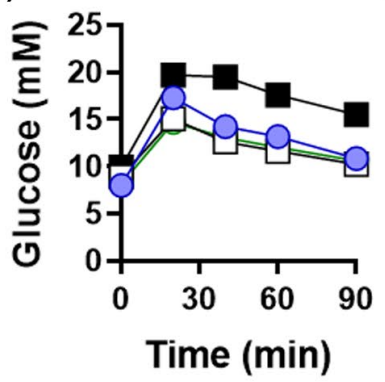

(J)
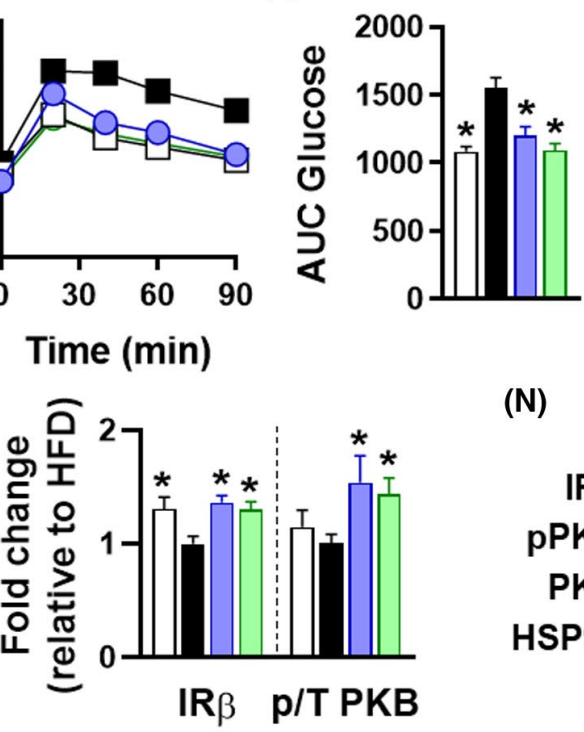

(O)
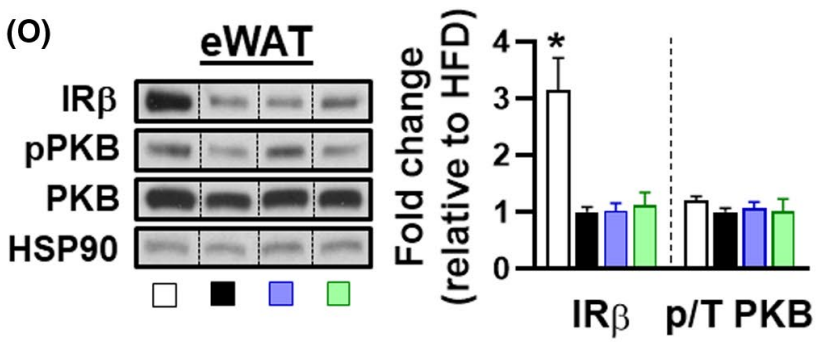

(C)

(N)

(P)
(F)

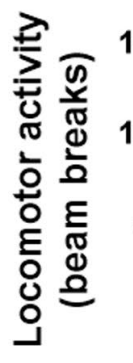

(K)

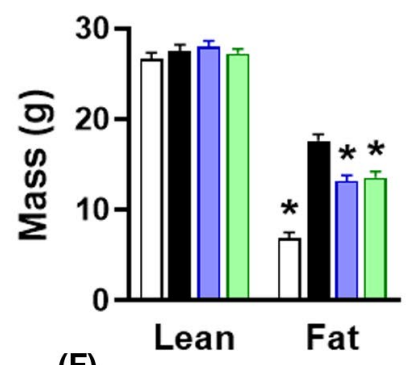

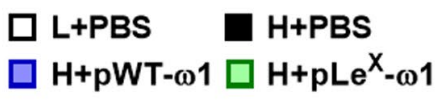

$\square \mathrm{H}+$ pWT- $\omega 1 \square \mathrm{H}+\mathrm{pLe}^{\mathrm{X}}-\omega 1$

(G)

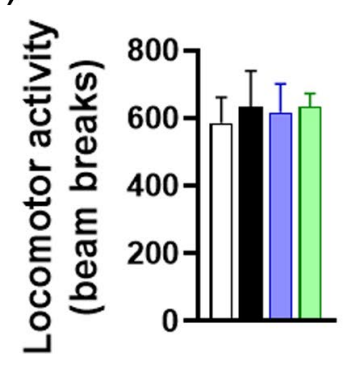

Time

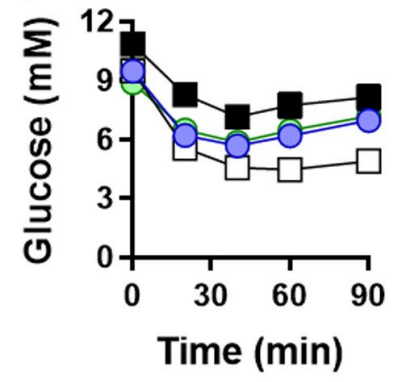

(L)

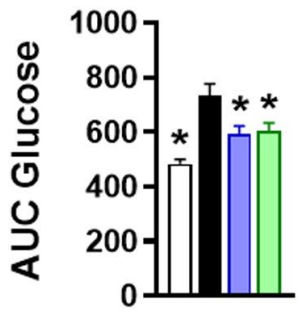

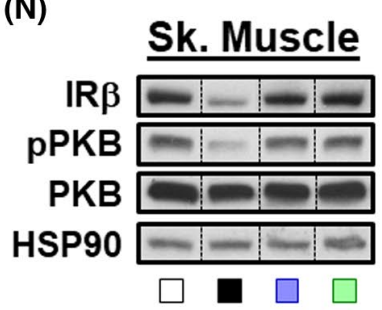
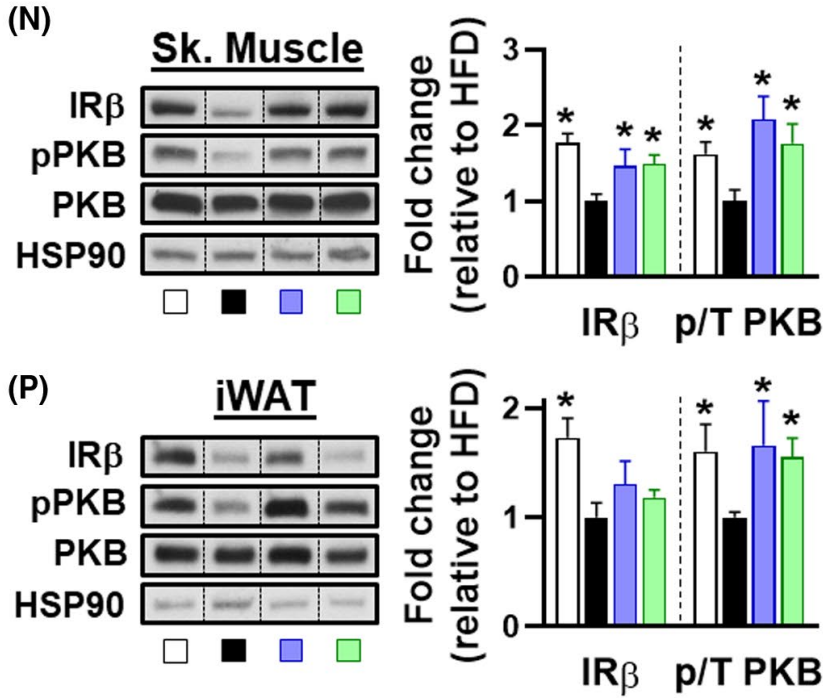
F I G U RE $3 \omega 1$ glycovariants reduce body weight, fat mass and food intake, and improve whole-body metabolic homeostasis in obese mice. Mice were fed a LFD (white bars) or a HFD for 12 weeks, and next received biweekly intraperitoneal injections of PBS (black bars) or $50 \mu \mathrm{g}$ pWT- $\omega 1$ (blue bars) or $\mathrm{pLe}^{\mathrm{X}}-\omega 1$ (green bars) during 4 weeks. Body weight (A and B) was monitored throughout the experimental period. Body composition (C) was measured after 4 weeks of treatment. Food intake (D and E) and locomotor activity (F and G) were assessed using fully automated single-housed metabolic cages during the first week of treatment. HOMA-IR at week $4(\mathrm{H})$ was calculated. Intraperitoneal glucose (I and $\mathrm{J}$ ) and insulin (K and L) tolerance tests were performed during week 3. Blood glucose levels were measured at the indicated time points (I and $\mathrm{K}$ ) and the AUC of the glucose excursion curve were calculated ( $\mathrm{J}$ and $\mathrm{L}$ ). After 4 weeks of treatment, mice received an i.p. injection of insulin (1 U/kg lean body mass) and after 15 minutes, pieces of liver (M), skeletal muscle (N), eWAT (O), and iWAT (P) were collected and snap-frozen immediately. The protein expression of IRb and the phosphorylation state of PKB-Ser473 (pPKB) were assessed by Western blot and quantified by densitometry analysis. HSP90 expression was used as internal housekeeping protein, and phosphorylation of PKB is expressed as a ratio of phosphorylated over total PKB. Representative Western blots are shown. Data shown are a pool of at least two independent experiments. Results are expressed as means \pm SEM. ${ }^{*} P<.05$ vs HFD $(n=11-20$ mice per group in A-C, H-L, and 4-9 mice per group in D-G, M-P)

glucose, plasma insulin levels (Figure S2E,F), and HOMA-IR (Figure $3 \mathrm{H}$ ) in obese mice, with a trend toward a stronger effect with $\mathrm{pLe}^{\mathrm{X}}-\omega 1$, indicating improved insulin sensitivity. Congruent with these data, we observed a significant improvement in whole-body glucose tolerance (Figure 3I,J) and insulin sensitivity (Figure 3K,L) in both pWT- $\omega 1$ and $\mathrm{pLe}^{\mathrm{X}}-$ $\omega 1$-treated obese mice. Furthermore, except in eWAT, the $\omega 1$ glycovariants restored the expression the insulin receptor (IRb) and the insulin-induced phosphorylation of PKB in the main metabolic organs, confirming enhanced insulin sensitivity (Figure 3M-P). In line with enhanced hepatic insulin sensitivity, we also found that pWT- $\omega 1$ and $p \mathrm{Pe}^{\mathrm{X}}-\omega 1$ lowered the glucose levels during an intraperitoneal pyruvate tolerance test (Figure S6J,K) and decreased the expression of gluconeogenic genes in the livers of obese mice (Figure S6L), suggesting an improved insulin-induced inhibition of hepatic gluconeogenesis.

Of note, the effects of $\omega 1$ glycovariants on food intake, plasma metabolic parameters, and whole-body insulin sensitivity were all dose-dependent (Figure S3) and already observed after 1 week of treatment, when body weight and fat mass were only mildly affected (Figure S4). Altogether, these data show that both recombinant $\omega 1$ glycovariants improve whole-body metabolic homeostasis in insulin-resistant obese mice.

\section{$3.4 \mid p_{L} e^{x}-\omega 1$ improves metabolic homeostasis in obese mice by a STAT6- independent mechanism}

We next investigated the role of type 2 immunity in the metabolic effects of $\omega 1$, using $\mathrm{pLe}^{\mathrm{X}}-\omega 1$ as the most potent and native-like glycovariant. As expected, while 4 weeks $\mathrm{pLe}^{\mathrm{X}}-\omega 1$ treatment (Figure 4A) increased WAT Th2 cells, eosinophils, and $\mathrm{YM}^{+}$AAMs in obese WT mice, this type 2 immune response was abrogated in obese Stat $^{-1}$ mice (Figure 4B-D). However, treatment with $\mathrm{pLe}^{\mathrm{X}}-\omega 1$ still reduced body weight (Figure 4E-G) and food intake (Figure $4 \mathrm{H}$ ), and affected body composition (Figure S2G) in Stat6 $^{-1-}$ obese mice to the same extent as in WT mice. In addition, both plasma insulin levels and HOMA-IR were markedly decreased in both genotypes (Figure S2H,I and Figure 4I). The improvements in whole-body glucose tolerance (Figure 4J-L) and insulin sensitivity (Figure 4M-O) were also still observed in $\mathrm{Stat}^{-/-}$mice, indicating that $\mathrm{pLe}^{\mathrm{X}}-\omega 1$ 's type 2 immunity-inducing capacity does not play a major role in restoration of metabolic homeostasis in obese mice. Of note, in contrast to its implication in maintenance of WAT metabolic homeostasis, IL-13 signaling has recently also been shown to play a role in the development of liver fibrosis. ${ }^{40,41}$ Interestingly, the increase in liver $\mathrm{IL}_{-} 5^{+}$and $\mathrm{IL}-13^{+}$Th2 cells in response to $\mathrm{pLe}^{\mathrm{X}}-\omega 1$ was also abrogated in Stat6 $^{-1-}$ mice (Figure S8E), and the expression of fibrotic gene markers were markedly reduced in Stat $^{-/-}$mice as compared to WT mice (Figure S8F). Taken together, these results show that $\mathrm{pLe}^{\mathrm{X}}-\mathrm{\omega} 1$ improves whole-body metabolic homeostasis independent of STAT6mediated type 2 immunity, while promoting early markers of hepatic fibrosis at least partly through an IL-13-STAT6mediated mechanism.

\section{5 | $\mathrm{pLe}^{\mathrm{X}}-\omega 1$ improves metabolic homeostasis through leptin receptor-independent inhibition of food intake in obese mice}

As $\omega 1$ significantly reduced food intake in obese mice, we next investigated its impact on feeding behavior. We found that a single intraperitoneal injection of $\mathrm{pLe}^{\mathrm{X}}-\omega 1$ in overnight-fasted obese mice markedly reduced food intake during refeeding for at least 24 hours, resulting in decreased body weight gain as compared to PBS-injected mice (Figure 5AC). To determine whether reduced food intake drives the beneficial metabolic effects of $\omega 1$, we treated HFD-fed mice with $\mathrm{pLe}^{\mathrm{X}}-\omega 1$ or PBS, and included a pair-fed group that received daily adjusted HFD meals based on the food intake of the $p L e^{\mathrm{X}}-\omega 1$-treated animals (Figure 5D). While $\mathrm{pLe}^{\mathrm{X}}-\omega 1$ induced IL- $13^{+}$Th 2 cells, eosinophils, and $\mathrm{YM} 1^{+} \mathrm{AAMs}$ in WAT, reducing caloric intake in pair-fed mice did not affect 
(A)

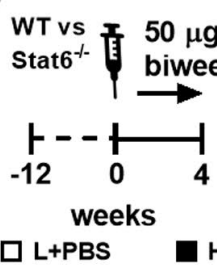

(E)

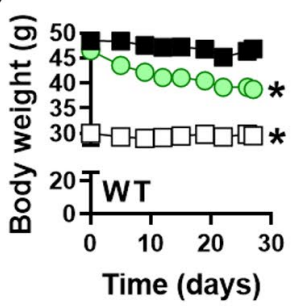

(J)

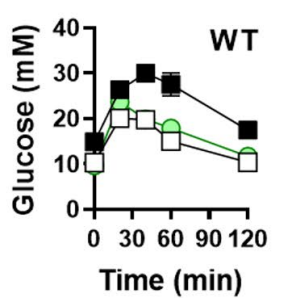

(K)
(B)

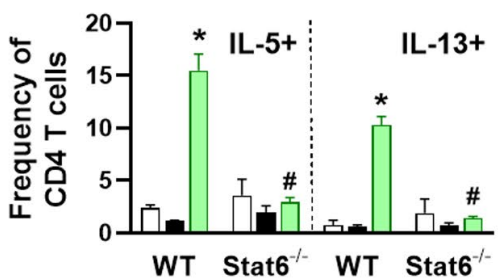

(F)

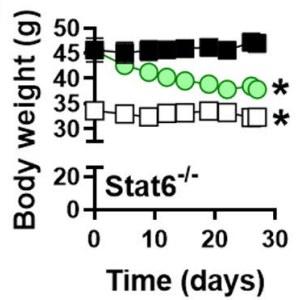

(C)

(G)

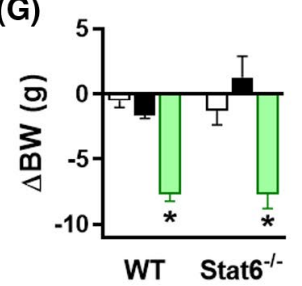

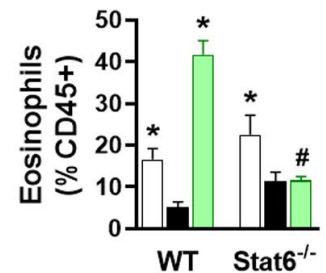

(H)

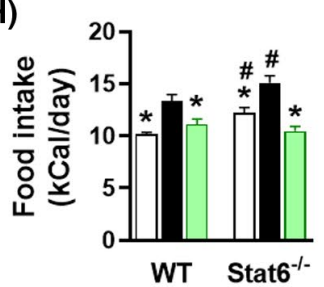

(D)

(M)
(L)
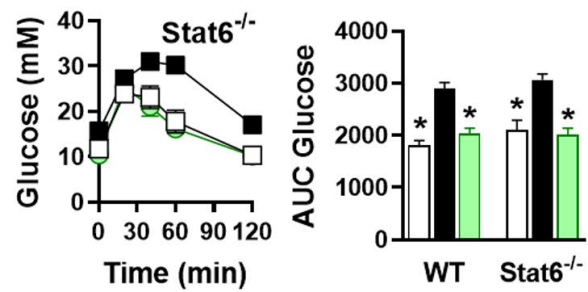

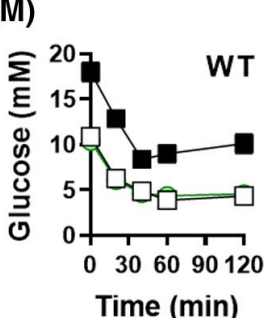

(N)

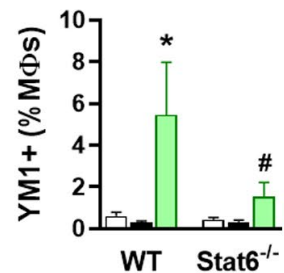

(l)

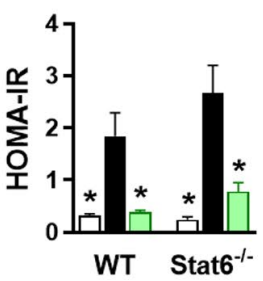

F I G U RE 4 pLe ${ }^{\mathrm{X}}-\omega 1$ improves metabolic homeostasis in obese mice by a STAT6-independent mechanism. WT and Stat $\sigma^{-/-}$mice were fed a LFD (white bars) or a HFD for 12 weeks and next received biweekly intraperitoneal injections of PBS (black bars) or $50 \mu \mathrm{g}$ pLe ${ }^{\mathrm{X}}$ - $\omega 1$ (green bars) during 4 weeks (A). At the end of the experiment, eWAT was collected, processed, and analyzed as described in the legend of Figure 1. The frequencies of cytokine-expressing CD4 T cells (B) were determined. Abundances of eosinophils (C) and YM1 ${ }^{+}$macrophages (D) were determined. Body weight (E-G) and food intake $(\mathrm{H})$ were monitored throughout the experimental period. HOMA-IR at week 4 (I) was calculated, and intraperitoneal glucose $(\mathrm{J}-\mathrm{L})$ and insulin $(\mathrm{M}-\mathrm{O})$ tolerance tests were performed as described in the legend of Figures 1 and 3. Results are expressed as means \pm SEM. ${ }^{*} P<.05$ vs HFD, ${ }^{\#} P<.05$ vs WT $(\mathrm{n}=3-5$ mice per group)

WAT type 2 immunity, as expected (Figure 5E-G). Yet, food restriction in the pair-fed group decreased body weight $5 \mathrm{H}$, fasting blood glucose and plasma insulin levels (Figure S2K,L), and HOMA-IR (Figure 5I) as well as whole-body glucose tolerance to the same extent as in $\mathrm{pLe}^{\mathrm{X}}$ - $\omega 1$-treated animals (Figure 5J,K).

The central regulation of feeding behavior and whole-body energy homeostasis involves complex neuronal networks, notably in the hypothalamus and brain stem. ${ }^{42,43}$ To investigate whether $\mathrm{pLe}^{\mathrm{X}}-\omega 1$ accumulates in the brain to directly regulate hypothalamic neurons controlling food intake, we performed in vivo imaging experiments with $\mathrm{pLe}^{\mathrm{X}}-\omega 1$ conjugated to a hybrid tracer $\left({ }^{111}\right.$ In-DTPA-Cy5-pLe $\left.{ }^{\mathrm{X}}-\omega 1\right)$. Both Single Photo Emission Computed Tomography (SPECT) imaging and radioactivity biodistribution revealed that ${ }^{111} \mathrm{In}$ DTPA-Cy5-pLe ${ }^{\mathrm{X}}-\omega 1$ mainly accumulated in abdominal organs, for example, adipose tissues, liver and intestines, and peritoneal draining lymph nodes, whereas no substantial amounts of radioactivity could be detected in the hypothalamus and other brain regions 24 hours after tracer administration (Figure S9A,B). Of note, both glycovariants did not affect HFD-induced expression of various inflammatory genes in the whole hypothalamus (Figure S9C), suggesting that dampening of hypothalamic inflammation is probably not involved in the anorexigenic effects of $\omega 1$. Hence, $\omega 1$ does not distribute to the brain and likely regulates food intake through peripheral effects.

The hypothalamus and brain stem also integrate signals from both the enteric nervous system and circulating hormones derived from adipose tissue and other peripheral tissues. Leptin is by far the best studied peripheral hormone that regulates food intake, increasing satiety by triggering STAT3-mediated pathways in the hypothalamic arcuate nucleus. $^{42}$ In order to study the role of leptin signaling in the metabolic effects of $\mathrm{pLe}^{\mathrm{X}}-\omega 1$, we used leptin receptor-deficient $d b / d b$ mice that are hyperphagic and naturally develop obesity and severe metabolic dysfunctions. ${ }^{44}$ In this model, $\mathrm{pLe}{ }^{\mathrm{X}}-\omega 1$ also increased WAT IL- $13^{+}$Th2 cells, eosinophils, and $\mathrm{YM}^{+}$AAMs (Figure 6A-D). Furthermore, $\mathrm{pLe}^{\mathrm{X}}-\omega 1$ still reduced body weight (Figure $6 \mathrm{E}, \mathrm{F}$ ), fat mass gain (Figure S2M,N), and food intake (Figure 6G), indicating that leptin signaling is not involved in the anorexigenic effect of $\omega 1$. Finally, plasma insulin levels (Figure S2P), HOMA-IR (Figure 6H), and whole-body glucose tolerance and insulin sensitivity (Figure 6I-L) were still significantly improved. 


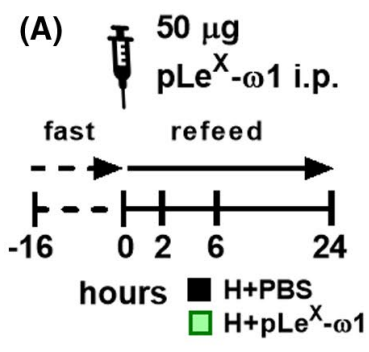

(E)

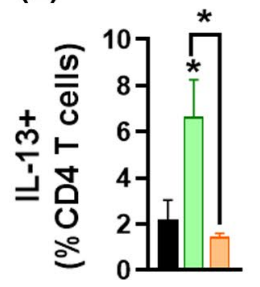

(B)

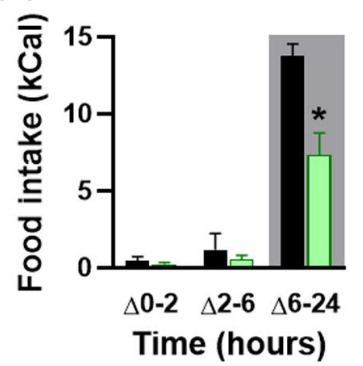

(F)

(G)

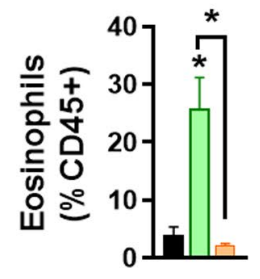

(C)

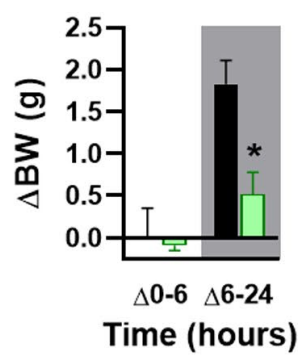

(I)

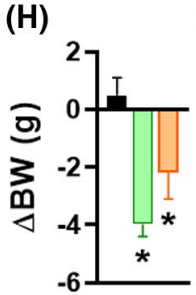

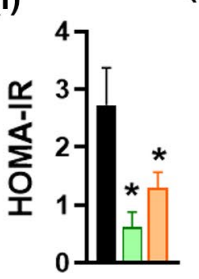

(D)

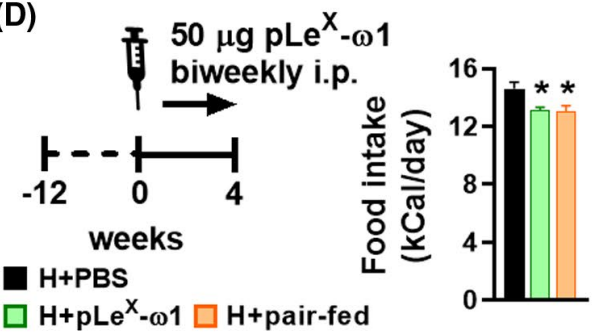

(J)

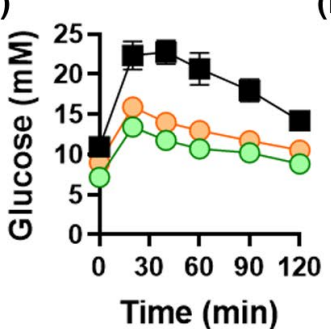

(K)

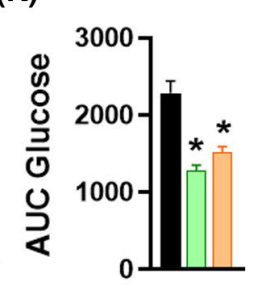

F I G U R E 5 pLe ${ }^{\mathrm{X}}-\omega 1$ inhibits fasting-induced refeeding and improves metabolic homeostasis through inhibition of food intake in obese mice. Mice were fed a HFD for 12 weeks and fasted overnight prior to intraperitoneal injections of either PBS (black bars) or $50 \mu \mathrm{g}$ pLe ${ }^{\mathrm{X}}$ - $\omega 1$ (green bars; A). Food intake (B) and body weight changes (C) were next monitored during 24 hours after refeeding. Mice were fed a HFD for 12 weeks, singlehoused, and next received biweekly intraperitoneal injections of PBS or $50 \mu \mathrm{g} \mathrm{pe}^{\mathrm{X}}-\omega 1$ during 4 weeks. In one group ( $\mathrm{H}+$ pair-fed; orange bars), the amount of food available for PBS-treated mice was adjusted daily in order to match the food intake of the pLe ${ }^{\mathrm{X}}$ - $\omega 1$-treated group (D). At the end of the experiment, eWAT was collected, processed, and analyzed as described in the legend of Figure 1. The frequencies of IL-13-expressing CD4 T cells (E), eosinophils (F), and YM1 ${ }^{+}$macrophages (G) were determined. Body weight change (H) was determined after 4 weeks. HOMA-IR (I) was calculated at week 4 and an i.p. glucose tolerance test ( $\mathrm{J}$ and $\mathrm{K}$ ) was performed at week 3, as described in the legend of Figure 1. Results are expressed as means \pm SEM. $* P<.05$ vs HFD or as indicated $(\mathrm{n}=3-5$ mice per group)

(A)

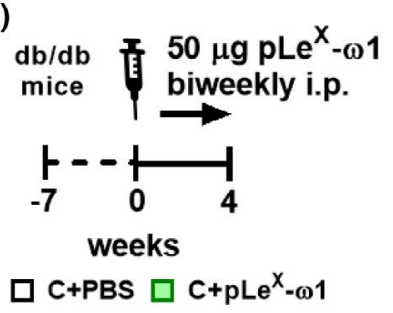

(B)

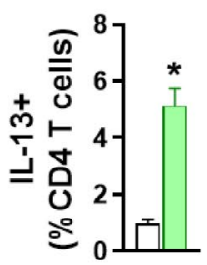

(C)

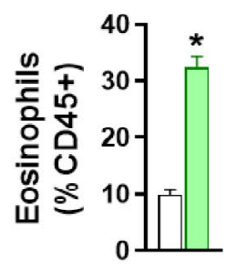

(D)

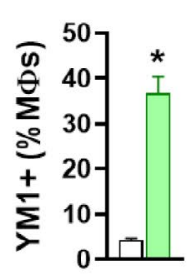

(E)

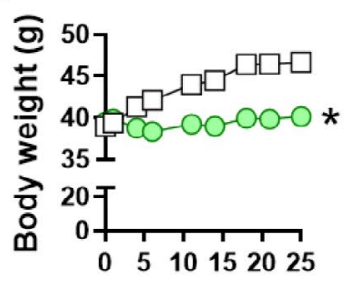

Time (days)

(L)

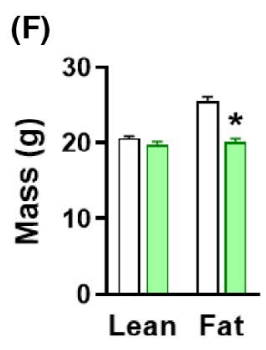

(G)

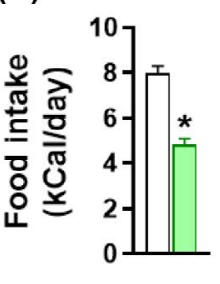

(H)

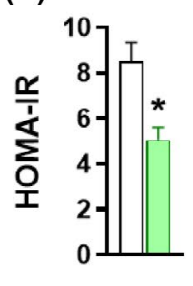

(I)

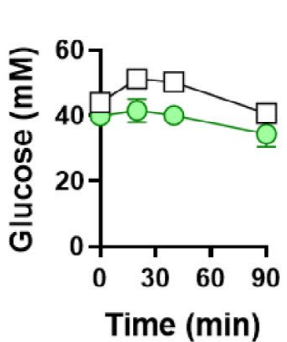

(K)

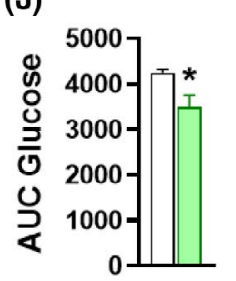

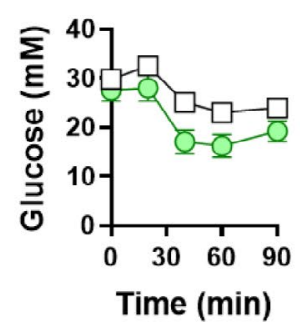

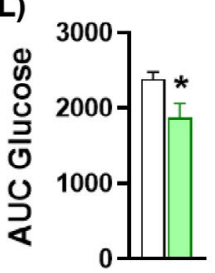

F I G URE 6 The metabolic effects of $\mathrm{pLe}^{\mathrm{X}}-\omega 1$ are independent of leptin signaling in hyperphagic obese mice. Seven weeks old obese $d b /$ $d b$ mice received biweekly intraperitoneal injections of PBS (white bars) or $50 \mu \mathrm{g} \mathrm{pLe}^{\mathrm{X}}$ - $\omega 1$ (green bars) during 4 weeks (A). At the end of the experiment, eWAT was collected, processed, and analyzed as described in the legend of Figure 1. The frequencies of IL-13-expressing CD4 T cells (B), eosinophils (C), and $\mathrm{YM1}^{+}$macrophages (D) were determined. Body weight (E) and food intake (G) were monitored throughout the experimental period, and body composition (F) was measured after 4 weeks. HOMA-IR (H) was calculated at week 4 and intraperitoneal glucose (I and $\mathrm{J})$ and insulin (K and $\mathrm{L}$ ) tolerance tests were performed as described in the legend of Figures 1 and 3. Results are expressed as means \pm SEM. $* P<.05$ vs PBS ( $\mathrm{n}=5-6$ mice per group) 
Collectively, our results show that $\omega 1$ improves wholebody metabolic homeostasis independent of its type $2 \mathrm{immu}-$ nity-inducing capacity, but by inhibiting food intake through a leptin receptor-independent mechanism.

\section{DISCUSSION}

Obesity-associated metaflammation promotes insulin resistance, while metabolic homeostasis is maintained by type 2 immunity. ${ }^{1}$ Since helminths are well known for inducing a potent type 2 immune response, their putative beneficial effects on insulin sensitivity and glucose homeostasis, together with the identification of specific helminth-derived molecules capable of driving such type 2 immune responses, have gained increasing attention..$^{11,16,45}$ The assumption has been that induction of type 2 immunity is the main mechanism by which helminths and helminth-derived molecules can improve metabolic homeostasis. The glycoprotein $\omega 1$, a T2 ribonuclease which is secreted from $S$ mansoni eggs, is one of the major immunomodulatory components in SEA and has previously been shown to condition DCs to prime Th2 responses, at least partly through its glycan-mediated uptake and intracellular RNase activity. ${ }^{17,18}$ Here, we report that two plant-produced recombinant $\omega 1$ glycovariants induced a rapid and sustained reduction in body weight and improved whole-body insulin sensitivity and glucose tolerance in obese mice. This improvement was associated with a strong type 2 immune response in WAT, characterized by a significant increase in Th2 cells, eosinophils, and AAMs. Contrary to SEA, $\omega 1$ still improved metabolic homeostasis in Stat6-deficient obese mice, indicating that its type $2 \mathrm{im}$ munity-inducing capacity does not play a major role. Indeed, we find that $\omega 1$ regulates energy consumption independent of leptin receptor signaling, which drives most of its metabolic effects. Altogether, these findings indicate that helminth-derived molecules may act through multiple distinct pathways for improving obesity-associated metabolic dysfunctions and further characterization of these molecules may lead to new therapeutic strategies for combating obesity.

A recent study from Hams et al also reported that acute treatment of HFD-fed obese mice with HEK-293-produced recombinant $\omega 1$-induced long-lasting weight loss, and improved glucose tolerance by a mechanism involving IL-33 and ILC2-mediated WAT type 2 immunity and adipose tissue beiging. ${ }^{19}$ In contrast to this report, while we also observed increased IL-33 mRNA expression in eWAT using the same $\omega 1$ concentrations (data not shown), we found no increase in WAT ILC2s after either 1, or 4 weeks of treatment with both plant-produced $\omega 1$ glycovariants. Moreover, we did not find evidence of WAT beiging in both eWAT and iWAT from obese mice. Finally, we also found that STAT6mediated type 2 immunity was dispensable for the metabolic effects of $\omega 1$. It should be noted that despite similar RNase activity when compared to native $\omega 1,{ }^{21}$ the recombinant $\omega 1$ produced by HEK-293 cells and the glyco-engineered molecules from $N$ benthamiana plants harbor significantly different $\mathrm{N}$-glycosylation patterns, ${ }^{18}$ which may partly explain the different outcomes between studies. Interestingly, as compared to pWT- $\omega 1$, we observed a trend for a stronger effect on insulin sensitivity and food intake with $\mathrm{pLe}^{\mathrm{X}}-\omega 1$, of which the glycans resemble the ones of native helminth $\omega 1$ the most.

In our study, both $\omega 1$ glycovariants were found to induce a type 2 immune response in WAT, characterized by a significant increase in Th2 cells, eosinophils, and AAMs. WAT eosinophilia is dependent on IL-5 and IL-13, which are predominantly expressed by ILC2s, and, to a lower extent, by Th2 cells in lean mice. ${ }^{9}$ As previously described for SEA, ${ }^{14}$ we showed that the $\omega 1$-induced increase in type 2 cytokines are clearly derived from $\mathrm{CD}^{+} \mathrm{T}$ cells, suggesting that $\mathrm{DC}-$ mediated Th2 skewing is required, rather than ILC2 activation, to induce WAT eosinophilia and AAM polarization. Of note, it was previously shown that $\mathrm{pLe}^{\mathrm{X}}-\omega 1$, compared to $\mathrm{pWT}-\omega 1$, induced a stronger Th2 polarization in vivo using a footpad immunization model in mice. ${ }^{21}$ In our conditions, both glycovariants induced a similar increase in the percentage of Th2 cells in metabolic tissues from obese mice, whereas $p \mathrm{pe}^{\mathrm{X}}-\omega 1$ increased total $\mathrm{CD} 4^{+} \mathrm{T}$ cells to a greater extent in the liver and to a lesser extent in WAT when compared to pWT- $\omega 1$. Altogether, this suggests that the different glycans on $\omega 1$ glycovariants might lead to tissue-specific targeting of $\omega 1$ and resulting differences in total Th2 cells.

While the type 2 immune response seems not to be significantly involved in the beneficial metabolic effects of $\omega 1$, we found that treatment with both $\omega 1$ glycovariants reduced food intake, with a trend for $\mathrm{pLe}^{\mathrm{X}}-\omega 1$ being more potent than pWT- $\omega 1$. This anorexigenic effect, which was not observed previously when mice were chronically infected with $S$ mansoni or treated with SEA [Figure 1F and Ref. 14], was dose-dependent and also observed in Stat6-deficient mice. Importantly, since both locomotor activity and lean body mass were not affected by $\omega 1$, this inhibition of food intake is unlikely to be caused by illnesses associated with catatonia. Using fast-refeeding and paired feeding experiments, we clearly showed that $\omega 1$ rapidly inhibited food intake, an effect that mainly contributed to the improvements in metabolic homeostasis. This inhibition of food intake persisted throughout the 4 weeks of treatment, indicating a long-lasting effect, and was already achieved by injecting $10 \mu \mathrm{g} \mathrm{pLe} \mathrm{X}^{\mathrm{X}}-\omega 1$ (data not shown). Of note, in the study from Hams et al using HEKproduced recombinant $\omega 1$ in the same concentration range as us, the effect of $\omega 1$ on feeding behavior and its putative contribution to the observed decrease in body weight and improvement of glucose tolerance in obese mice have not been specifically investigated. ${ }^{19}$ 
Anorexia is one of the clinical manifestations of infection with different helminth species in both animals and humans. As such, deworming children infected with the hookworms Ascaris lumbricoides and/or Trichuris trichiura has been reported to increase appetite, ${ }^{46}$ suggesting a relationship between helminth infection and food intake. In rodents, infection with Taenia taeniaeformis and Nippostrongylus brasiliensis both induced anorexia by modulating neuropeptide expression in the hypothalamus, ${ }^{47,48}$ indicating that helminths and/or helminth products may regulate feeding behavior. The mechanism by which $\omega 1$ inhibits food intake is, however, still unknown and will require further neuroscience-driven approaches to be elucidated. Regulation of food intake by the central nervous system is a complex process involving both local and peripheral neuro-immuno-endocrine inputs that are mainly integrated in the hypothalamic arcuate nucleus and the brain stem nucleus tractus solitarius. ${ }^{42,43}$ In our study, we did not detect accumulation of radioactively labeled $\omega 1$ in the brain 24 hours after intraperitoneal injection, suggesting that the glycoprotein may exerts its anorexigenic effects via peripheral rather than central action(s). Upon meal ingestion, several anorexigenic peptides and hormones are produced by metabolic organs, including adipose tissues and the intestine, and can either directly act on specific neurons after crossing the blood-brain barrier or signal from the periphery via vagal nerve-mediated pathways that contribute to satiety regulation. ${ }^{49,50}$ Leptin is a key adipose tissue-derived anorexigenic hormone which signals through the leptin receptor expressed in specific neurons located in the arcuate nucleus of the hypothalamus to reduce food intake. ${ }^{42}$ During obesity, hypothalamic inflammation has been reported to impair whole-body energy homeostasis, at least partly by inducing central leptin resistance and subsequent increased food intake. ${ }^{51-53}$ However, despite some evidence of improved systemic leptin sensitivity by $\omega 1$ (data not shown), we showed that the hypothalamic expression of key inflammatory genes were not affected by $\omega 1$ in obese mice, suggesting that modulation of hypothalamic inflammation is likely not involved in its anorexigenic effect. Furthermore, we found that its anorexigenic and metabolic effects were still present in leptin receptor-deficient mice, allowing us to exclude a significant contribution of peripheral/central leptin signaling. Among the peripheral signals that regulate feeding behavior, it would be interesting to explore the involvement of a gut-brain axis, potentially secondary to changes in gut microbiota, through vagal nerve ablation. ${ }^{50}$ Recently, $N$ brasiliensis infection and its products were also shown to increase production of the neuropeptide Neuromedin $U$ by mucosal neurons, allowing the host to mount an effective type 2 immune response. ${ }^{54-56}$ Neuromedin $\mathrm{U}$ also has anorexigenic effects, ${ }^{57}$ thus, it is tempting to speculate that some helminth molecules may indirectly trigger anorexia through neuro-immune interactions in the gut.
It is worth mentioning that $\omega 1$ also increased IL-13 producing Th2 cells in the liver, but, unlike SEA, ${ }^{14}$ promoted CD11c expression in Kupffer cells while not affecting the expression of YM1, suggesting that macrophages are rather polarized toward a pro-inflammatory state in this tissue. An increase in hepatic expression of fibrotic gene markers and circulating ALAT levels was also observed, both indicating increased liver damage induced by $\omega 1$. Interestingly, the $\omega 1$-induced increase in IL-13 ${ }^{+}$Th2 cells and IL-13 gene expression in the liver were markedly reduced in Stat6-deficient mice, which was accompanied by a decreased expression of fibrotic gene markers. Collectively, these findings confirmed previous studies describing that IL-13 plays a role in the development of liver fibrosis, ${ }^{40,41}$ and that $\omega 1$ has cytotoxic effects in the liver. ${ }^{38,39}$

In conclusion, we report here that the helminth glycoprotein $\omega 1$ improved metabolic homeostasis in insulin-resistant obese mice independent of its type 2 immunity-inducing capacity. Rather, the $\omega 1$-induced metabolic improvements in obese mice were mostly attributable to leptin receptor-independent inhibition of food intake. Further studies are required to unravel such underlying mechanisms, notably exploring the role of gut hormones on peripheral and/or central regulation of feeding behavior. Of note, with regards to its putative therapeutic potential for metabolic disorders, it is important to underline that despite beneficial effects on whole-body metabolic homeostasis, $\omega 1$ also induced early markers of mild hepatic fibrosis, partly through a type 2 immunity-mediated mechanism. Finally, by contrast to $\omega 1$, the complex mixture of SEA does not have detrimental effects on the liver and improves metabolic homeostasis through a STAT6-mediated type 2 immune response, suggesting that it may contain some other unidentified molecules, such as Dectin 2 ligands, ${ }^{58}$ with potentially beneficial immunometabolic properties.

\section{ACKNOWLEDGMENTS}

The authors thank Gerard van der Zon and Tessa Buckle (Leiden University Medical Center, Leiden, the Netherlands), and Uma Mahesh Gundra, Ada Weinstock, Jian-Da Lin, and Mei San Tang (New York University School of Medicine, New York, USA) for their invaluable technical assistance. The authors also thank Ko Willems van Dijk and Patrick Rensen (Leiden University Medical Center) for allowing the use of the LUMC metabolic phenotyping platform (MRI and metabolic cages). This study was supported by Dutch Organization for Scientific Research (NWO; ZonMW TOP Grant 91214131 to $\mathrm{CH}$, AS, MY, and BG) and the NWO Graduate School Program (022.006.010 to HvdZ). This work was also supported in part by the Division of Intramural Research, National Institutes of Health (NIH)/National Institute of Allergy and Infectious Diseases (NIAID) and Awards AI130945 and AI133977, NIH/National Heart, Lung, and Blood Institute 
Award HL084312, and US Department of Defense Award W81XWH-16-1-0256 (to PL). The funders had no role in study design, data collection and analysis, decision to publish, or preparation of the manuscript.

\section{CONFLICT OF INTEREST}

The authors have stated there are no conflicts of interest in connection with this article.

\section{AUTHOR CONTRIBUTIONS}

HJP van der Zande, MA Gonzalez, CH Hokke, M. Yazdanbakhsh, P. Loke, and B. Guigas conceptualized research; HJP van der Zande, M. van Huizen, J. Poles, and B. Guigas analyzed data; HJP van der Zande, MA Gonzalez, K. de Ruiter, N. García-Tardón, M. van Huizen, LR Pelgrom, JM Lambooij, A. Zawistowska-Deniziak, and M. Welling performed research; RHP Wilbers, K. van Noort, D. van Willigen, M. Welling, F. van Leeuwen, and A. Schots provided resources; and HJP van der Zande, K. de Ruiter and B. Guigas wrote the manuscript.

\section{REFERENCES}

1. Donath MY, Shoelson SE. Type 2 diabetes as an inflammatory disease. Nat Rev Immunol. 2011;11(2):98-107.

2. Lackey DE, Olefsky JM. Regulation of metabolism by the innate immune system. Nat Rev Endocrinol. 2016;12(1):15-28.

3. Kolb H, Mandrup-Poulsen T. The global diabetes epidemic as a consequence of lifestyle-induced low-grade inflammation. Diabetologia. 2010;53(1):10-20.

4. Lumeng CN, DelProposto JB, Westcott DJ, Saltiel AR. Phenotypic switching of adipose tissue macrophages with obesity is generated by spatiotemporal differences in macrophage subtypes. Diabetes. 2008;57(12):3239-3246.

5. Obstfeld AE, Sugaru E, Thearle M, et al. C-C chemokine receptor 2 (CCR2) regulates the hepatic recruitment of myeloid cells that promote obesity-induced hepatic steatosis. Diabetes. 2010;59(4):916-925.

6. Kratz M, Coats B, Hisert K, et al. Metabolic dysfunction drives a mechanistically distinct proinflammatory phenotype in adipose tissue macrophages. Cell Metab. 2014;20(4):614-625.

7. Lanthier N, Molendi-Coste O, Horsmans Y, van Rooijen N, Cani PD, Leclercq IA. Kupffer cell activation is a causal factor for hepatic insulin resistance. Am J Physiol Gastrointest Liver Physiol. 2010;298(1):G107-G116.

8. Talukdar S, Oh DY, Bandyopadhyay G, et al. Neutrophils mediate insulin resistance in mice fed a high-fat diet through secreted elastase. Nat Med. 2012;18(9):1407-1412.

9. Molofsky AB, Nussbaum JC, Liang H-E, et al. Innate lymphoid type 2 cells sustain visceral adipose tissue eosinophils and alternatively activated macrophages. J Exp Med. 2013;210(3):535-549.

10. Wu D, Molofsky AB, Liang H-E, et al. Eosinophils sustain adipose alternatively activated macrophages associated with glucose homeostasis. Science. 2011;332(6026):243-247.

11. van der Zande HJP, Zawistowska-Deniziak A, Guigas B. Immune regulation of metabolic homeostasis by helminths and their molecules. Trends Parasitol. 2019;35(10):795-808.
12. Maizels RM, Yazdanbakhsh M. Immune regulation by helminth parasites: cellular and molecular mechanisms. Nat Rev Immunol. 2003;3(9):733-744.

13. Zinsou JF, Janse JJ, Honpkehedji YY, et al. Schistosoma haematobium infection is associated with lower serum cholesterol levels and improved lipid profile in overweight/obese individuals. PLoS Negl Trop Dis. 2020;14(7):e0008464.

14. Hussaarts L, García-Tardón N, Beek L, et al. Chronic helminth infection and helminth-derived egg antigens promote adipose tissue M2 macrophages and improve insulin sensitivity in obese mice. FASEB J. 2015;29(7):3027-3039.

15. Okano M, Satoskar AR, Nishizaki K, Abe M, Harn DA Jr. Induction of Th2 responses and $\mathrm{IgE}$ is largely due to carbohydrates functioning as adjuvants on Schistosoma mansoni egg antigens. J Immunol. 1999;163(12):6712-6717.

16. Hussaarts L, Yazdanbakhsh M, Guigas B. Priming dendritic cells for th2 polarization: lessons learned from helminths and implications for metabolic disorders. Front Immunol. 2014;5:499.

17. Everts B, Perona-Wright G, Smits HH, et al. Omega-1, a glycoprotein secreted by Schistosoma mansoni eggs, drives Th2 responses. J Exp Med. 2009;206(8):1673-1680.

18. Everts B, Hussaarts L, Driessen NN, et al. Schistosome-derived omega-1 drives Th2 polarization by suppressing protein synthesis following internalization by the mannose receptor. J Exp Med. 2012;209(10):1753-1767.

19. Hams E, Bermingham R, Wurlod FA, et al. The helminth T2 RNase omega1 promotes metabolic homeostasis in an IL-33- and group 2 innate lymphoid cell-dependent mechanism. FASEB J. 2016;30(2):824-835.

20. Meevissen MH, Wuhrer M, Doenhoff MJ, et al. Structural characterization of glycans on omega-1, a major Schistosoma mansoni egg glycoprotein that drives Th2 responses. J Proteome Res. 2010;9(5):2630-2642.

21. Wilbers RHP, Westerhof LB, van Noort K, et al. Production and glyco-engineering of immunomodulatory helminth glycoproteins in plants. Sci Rep. 2017;7:45910.

22. Grogan JL, Kremsner PG, Deelder AM, Yazdanbakhsh M. Elevated proliferation and interleukin-4 release from CD4+ cells after chemotherapy in human Schistosoma haematobium infection. Eur J Immunol. 1996;26(6):1365-1370.

23. Lee S, Muniyappa R, Yan XU, et al. Comparison between surrogate indexes of insulin sensitivity and resistance and hyperinsulinemic euglycemic clamp estimates in mice. Am J Physiol Endocrinol Metab. 2008;294(2):E261-E270.

24. Stephenne X, Foretz M, Taleux N, et al. Metformin activates AMPactivated protein kinase in primary human hepatocytes by decreasing cellular energy status. Diabetologia. 2011;54(12):3101-3110.

25. Gundra UM, Girgis NM, Gonzalez MA, et al. Vitamin A mediates conversion of monocyte-derived macrophages into tissue-resident macrophages during alternative activation. Nat Immunol. 2017;18(6):642-653.

26. Hashimshony T, Senderovich N, Avital G, et al. CEL-Seq2: sensitive highly-multiplexed single-cell RNA-Seq. Genome Biol. 2016;17:77.

27. Langmead B, Salzberg SL. Fast gapped-read alignment with Bowtie 2. Nat Methods. 2012;9(4):357-359.

28. Anders S, Pyl PT, Huber W. HTSeq-a Python framework to work with high-throughput sequencing data. Bioinformatics. 2015;31(2):166-169. 
29. Thomas A, Belaidi E, Aron-Wisnewsky J, et al. Hypoxia-inducible factor prolyl hydroxylase 1 (PHD1) deficiency promotes hepatic steatosis and liver-specific insulin resistance in mice. Sci Rep. 2016;6:24618.

30. Kleiner DE, Brunt EM, Van Natta M, et al. Design and validation of a histological scoring system for nonalcoholic fatty liver disease. Hepatology. 2005;41(6):1313-1321.

31. Hensbergen AW, van Willigen DM, Welling MM, et al. Click chemistry in the design and production of hybrid tracers. ACS Omega. 2019;4(7):12438-12448.

32. Spa SJ, Welling MM, van Oosterom MN, et al. A supramolecular approach for liver radioembolization. Theranostics. 2018;8(9):2377-2386.

33. Takeda K, Tanaka T, Shi W, et al. Essential role of Stat6 in IL-4 signalling. Nature. 1996;380(6575):627-630.

34. Takeda K, Kamanaka M, Tanaka T, Kishimoto T, Akira S. Impaired IL-13-mediated functions of macrophages in STAT6-deficient mice. J Immunol. 1996;157(8):3220-3222.

35. Bhargava $\mathrm{P}, \mathrm{Li} \mathrm{C}$, Stanya KJ, et al. Immunomodulatory glycan LNFPIII alleviates hepatosteatosis and insulin resistance through direct and indirect control of metabolic pathways. Nat Med. 2012;18(11):1665-1672.

36. Van den Bossche J, O'Neill LA, Menon D. Macrophage immunometabolism: where are we (going)? Trends Immunol. 2017;38(6):395-406.

37. Ricardo-Gonzalez RR, Red Eagle A, Odegaard JI, et al. IL-4/STAT6 immune axis regulates peripheral nutrient metabolism and insulin sensitivity. Proc Natl Acad Sci U S A. 2010;107(52):22617-22622.

38. Dunne DW, Lucas S, Bickle Q, et al. Identification and partial purification of an antigen (omega 1) from Schistosoma mansoni eggs which is putatively hepatotoxic in T-cell deprived mice. Trans $R$ Soc Trop Med Hyg. 1981;75(1):54-71.

39. Abdulla MH, Lim KC, McKerrow JH, Caffrey CR. Proteomic identification of IPSE/alpha-1 as a major hepatotoxin secreted by Schistosoma mansoni eggs. PLoS Negl Trop Dis. 2011;5(10):e1368.

40. Gieseck R, Ramalingam T, Hart K, et al. Interleukin-13 Activates Distinct Cellular Pathways Leading to Ductular Reaction, Steatosis, and Fibrosis. Immunity. 2016;45(1):145-158.

41. Hart KM, Fabre T, Sciurba JC, et al. Type 2 immunity is protective in metabolic disease but exacerbates NAFLD collaboratively with TGF-beta. Sci Transl Med. 2017;9(396).

42. Schwartz MW, Woods SC, Porte D Jr, Seeley RJ, Baskin DG. Central nervous system control of food intake. Nature. 2000;404(6778):661-671.

43. Schneeberger M, Gomis R, Claret M. Hypothalamic and brainstem neuronal circuits controlling homeostatic energy balance. $J$ Endocrinol. 2014;220(2):T25-46.

44. Hummel KP, Dickie MM, Coleman DL. Diabetes, a new mutation in the mouse. Science. 1966;153(3740):1127-1128.

45. de Ruiter K, Tahapary DL, Sartono E, et al. Helminths, hygiene hypothesis and type 2 diabetes. Parasite Immunol. 2017;39(5).

46. Hadju V, Stephenson LS, Abadi K, Mohammed HO, Bowman DD, Parker RS. Improvements in appetite and growth in helminth-infected schoolboys three and seven weeks after a single dose of pyrantel pamoate. Parasitology. 1996;113(Pt 5):497-504.

47. Lohmus M, Moalem S, Bjorklund M. Leptin, a tool of parasites? Biol Lett. 2012;8(5):849-852.

48. Horbury SR, Mercer JG, Chappell LH. Anorexia induced by the parasitic nematode, Nippostrongylus brasiliensis: effects on NPY and CRF gene expression in the rat hypothalamus. J Neuroendocrinol. 1995;7(11):867-873.

49. Murphy KG, Bloom SR. Gut hormones and the regulation of energy homeostasis. Nature. 2006;444(7121):854-859.

50. Li Z, Yi C-X, Katiraei S, et al. Butyrate reduces appetite and activates brown adipose tissue via the gut-brain neural circuit. Gut. 2018;67(7):1269-1279.

51. Cai D, Liu T. Hypothalamic inflammation: a double-edged sword to nutritional diseases. Ann N Y Acad Sci. 2011;1243:E1-39.

52. Valdearcos M, Douglass JD, Robblee MM, et al. Microglial inflammatory signaling orchestrates the hypothalamic immune response to dietary excess and mediates obesity susceptibility. Cell Metab. 2017;26(1):185-97 e3.

53. Lee CH, Kim HJ, Lee Y-S, et al. Hypothalamic macrophage inducible nitric oxide synthase mediates obesity-associated hypothalamic inflammation. Cell Rep. 2018;25(4):934-46 e5.

54. Cardoso V, Chesné J, Ribeiro H, et al. Neuronal regulation of type 2 innate lymphoid cells via neuromedin U. Nature. 2017;549(7671):277-281.

55. Klose CSN, Mahlakõiv T, Moeller JB, et al. The neuropeptide neuromedin U stimulates innate lymphoid cells and type 2 inflammation. Nature. 2017;549(7671):282-286.

56. Wallrapp A, Riesenfeld SJ, Burkett PR, et al. The neuropeptide NMU amplifies ILC2-driven allergic lung inflammation. Nature. 2017;549(7672):351-356.

57. Hanada R, Teranishi H, Pearson JT, et al. Neuromedin U has a novel anorexigenic effect independent of the leptin signaling pathway. Nat Med. 2004;10(10):1067-1073.

58. Kaisar MMM, Ritter M, del Fresno C, et al. Dectin-1/2-induced autocrine PGE2 signaling licenses dendritic cells to prime Th2 responses. PLoS Biol. 2018;16(4):e2005504.

\section{SUPPORTING INFORMATION}

Additional supporting information may be found online in the Supporting Information section.

How to cite this article: van der Zande HJP, Gonzalez MA, de Ruiter K, et al. The helminth glycoprotein omega-1 improves metabolic homeostasis in obese mice through type 2 immunityindependent inhibition of food intake. The FASEB Journal. 2021;35:e21331. https://doi.org/10.1096/ fj.202001973R 